\title{
Microbiota links to neural dynamics supporting threat processing
}

\author{
Caitlin V. Hall $^{1,2,3}$ @ | Ben J. Harrison ${ }^{4}$ | Kartik K. Iyer ${ }^{1}$ | Hannah S. Savage ${ }^{4}$ | \\ Martha Zakrzewski $^{5}$ | Lisa A. Simms ${ }^{5}$ | Graham Radford-Smith ${ }^{5}$ | \\ Rosalyn J. Moran ${ }^{2}$ | Luca Cocchi ${ }^{1,3}$
}

${ }^{1}$ Clinical Brain Networks Group, QIMR Berghofer Medical Research Institute, Brisbane, Queensland, Australia

${ }^{2}$ Department of Neuroimaging, Institute of Psychiatry, Psychology \& Neuroscience, Kings College London, London, UK

${ }^{3}$ School of Biomedical Sciences, Faculty of Medicine, University of Queensland, Brisbane, Queensland, Australia

${ }^{4}$ Melbourne Neuropsychiatry Centre, Department of Psychiatry, The University of Melbourne \& Melbourne Health, Melbourne, Victoria, Australia

${ }^{5}$ Gut Health LAB, QIMR Berghofer Medical Research Institute, Brisbane, Queensland, Australia

\section{Correspondence}

Caitlin V. Hall and Luca Cocchi, Clinical Brain Networks Group, QIMR Berghofer Medical Research Institute, 300 Herston Road, Brisbane, Queensland 4006, Australia. Email: caitlin.hall@qimrberghofer.edu.au and luca.cocchi@qimrberghofer.edu.au

\section{Funding information}

National Health and Medical Research Council, Grant/Award Number: APP1099082

\begin{abstract}
There is growing recognition that the composition of the gut microbiota influences behaviour, including responses to threat. The cognitive-interoceptive appraisal of threat-related stimuli relies on dynamic neural computations between the anterior insular (AIC) and the dorsal anterior cingulate ( $\mathrm{AACC}$ ) cortices. If, to what extent, and how microbial consortia influence the activity of this cortical threat processing circuitry is unclear. We addressed this question by combining a threat processing task, neuroimaging, 16S rRNA profiling and computational modelling in healthy participants. Results showed interactions between high-level ecological indices with threatrelated AIC-dACC neural dynamics. At finer taxonomic resolutions, the abundance of Ruminococcus was differentially linked to connectivity between, and activity within the AIC and $\mathrm{AACC}$ during threat updating. Functional inference analysis provides a strong rationale to motivate future investigations of microbiota-derived metabolites in the observed relationship with threat-related brain processes.
\end{abstract}

\section{KEYWORDS}

anterior insula, dorsal anterior cingulate, gut-brain axis, microbiota, neuroimaging, threat processing

\section{1 | INTRODUCTION}

Emerging work suggests that the composition and diversity of the intestinal (gut) microbiota plays a key role in altering brain activity and related behaviour (Cryan and Dinan, 2012). Microbiota-deficient and germ-free mice have provided initial accounts of the effect of microbiota on brain processes, including emotion and affect (Bravo et al., 2011), social behaviour (Sherwin et al., 2019), and cognition (Desbonnet et al., 2015). By extension, there is growing motivation to assess how microbial perturbations are linked to the expression of psychiatric symptoms including the ability to protect against (Clarke et al., 2013), or induce (Neufeld et al., 2011) stress and anxiety-like behaviour.

A key feature underpinning anxiety is the impaired ability to flexibly respond to threat and modify behavioural responses in volatile learning environments (Schiller et al., 2008). Within a broader network of cortico-subcortical regions, the anterior insular cortex (AIC) and dorsal anterior cingulate cortex (dACC) have been the most consistently implicated regions in neuroimaging studies of human threat processing (Fullana et al., 2016). In this capacity, the AIC-dACC 
circuitry has been hypothesised to support the subjective experience of threat processing via cognitive-interoceptive appraisal mechanisms (Kalisch and Gerlicher, 2014; Harrison et al., 2015; Fullana et al., 2016). Specifically, the AIC is thought to be responsible for generating an awareness of the current emotional and internal physical state (Craig, 2009; Fullana et al., 2016), including gastrointestinal and cardiorespiratory bodily changes (Garfinkel et al., 2015; Rebollo et al., 2018). This information is relayed to the dACC, where its activity has been more directly linked to the cognitive appraisal of bodily sensations of anxiety (Harrison et al., 2015). Given their joint contribution in receiving and processing afferent visceral information, the AIC-dACC network represents a natural candidate to study gut microbiota interactions with higher-level brain function (Kano et al., 2018).

Bidirectional communication between the microbiota and the brain is facilitated by several mechanisms, including immune, endocrine, vagal and microbiota-derived metabolite signalling (Raybould, 2010; Mayer, 2011; Cryan and Dinan, 2012; Kaelberer et al., 2018). Microbial-derived metabolites like short-chain fatty acids (SCFAs) can activate receptors expressed on the colonic epithelium and within enteroendocrine cells, modulating vagal afferents or immunomodulatory pathways (Le Poul et al., 2003; Koh et al., 2016). Vagal signalling may represent the most direct route through which the microbiota can relay action potentials to cortical regions involved in threat processing (Rhee et al., 2009; Raybould, 2010; Forsythe et al., 2014; Kaelberer et al., 2018). The latency of onset to evoking afferent vagal responses occurs within minutes of intraluminal probiotic administration (Perez-Burgos et al., 2012), while the SCFA butyrate-a by-product of microbial metabolism-has been shown to elicit vagal responses within seconds (Lal et al., 2001). Afferent viscerosensory signals converge in the nucleus tractus solitarius (NTS) and are projected via nuclei in the brainstem-including locus coeruleus, parabrachial nucleus and dorsal raphe nuclei-to higher cortical regions, including the AIC and dACC (Azzalini et al., 2019). The proposed relationship between the viscera and the AIC-dACC network is also supported by the cellular substrate underpinning communication between these two cortical regions: the von Economo neurons (VEN; Mayer, 2011). These cells contain receptors (serotonin $2 \mathrm{~b}$ ) and peptides (neuromedin B) that are also abundant in the enteric nervous system (Allman et al., 2010). The peculiar expression of receptors and peptides in both viscera and VENs suggest a likely role of the AIC-dACC network in linking brain and gut signals.

Preclinical work has revealed how manipulation of the microbiota impacts threat-related processes (Chu et al., 2019). These works provide preliminary support for the notion that changes in the composition of the microbiota, and associated metabolite production, modulates neural activity in distinct brain regions. However, due to the large variability between the mouse and human microbiota, preclinical observations have not always replicated in human studies (Sherwin et al., 2019). It is also unclear whether human microbiotabrain interactions can be characterised by shifts in high-level ecological measures (e.g. alpha diversity), or whether they emerge within taxonomic scales at finer resolutions. To bridge this knowledge gap, we combined a threat processing paradigm (Savage et al., 2020a;
Figure 1a), 16S rRNA gene sequencing (of stool samples), functional magnetic resonance imaging (fMRI) and computational modelling. Using established conditioning procedures, the adopted task facilitates the assessment of both general threat learning as well as threat updating responses in AIC and dACC. Recently, using this task, it was shown that threat updating responses in $\mathrm{AACC}$ were especially predictive of subjectively reported bodily anxiety sensations (Savage et al., 2020a).

Our first aim was to extend existing neuroimaging work by demonstrating the effective (causal) connectivity patterns between neural populations within the AIC and the $\mathrm{AACC}$ during the assessment of general threat acquisition, and subsequent updating processes. To understand whether inter-individual differences in microbiota covaried with evoked AIC-dACC responses during threat processing, we adopted a Bayesian and multivariate statistical framework (Zeidman et al., 2019). Specifically, effective connectivity measures were assessed against (i) high-level ecological measures ( $\alpha$-diversity and phylum ratio) and (ii) specific (genus-level) measures to discern the resolution/s at which interactions emerge. High-level microbial measures were selected a priori based on previous work (Ley et al., 2006; Mariat et al., 2009; Lozupone et al., 2012). At genus-level, we used a validated data-driven clustering approach (Arumugam et al., 2011) to discern features contributing to the largest variance among samples. The selection of genus-level candidates were consistent with previous literature (Tillisch et al., 2017; VallesColomer et al., 2019) and were assessed both independently (multiple regressions) and in the context of a multivariate microbiome-brain relationship (sparse canonical correlation analysis). Finally, guided by preclinical work, we assessed the predicted functional capacity of the microbiota-focusing on pathways involved in SCFA production. This last analysis aimed to provide support to previously proposed causal pathways linking microbiota to brain (van de Wouw et al., 2018; Boehme et al., 2019; Lee et al., 2020).

\section{2 | MATERIALS AND METHODS}

\section{1 | Participants}

The study was approved by the Human Research Ethics Committee of QIMR Berghofer Medical Research Institute (P3435). Written informed consent was obtained for all participants. Thirty-eight healthy adult participants (31.7 \pm 8.8 years; 23 female) were recruited from the Brisbane (Australia) metropolitan area by an accredited practising dietitian (CVH; Table S4). Exclusion criteria included: a BMI of $<18.5$ or > 30.0; current or previous history of a major psychiatric illness or neurological disorder (assessed via the Mini-International Neuropsychiatric Interview [Sheehan et al., 1998] performed by a trained psychologist); chronic or clinically significant pulmonary, cardiovascular, gastrointestinal, hepatic, renal, or dermatological functional abnormality as determined by medical history; history of cancer (excluding medically managed squamous or basal cell carcinomas of the skin); history of active, uncontrolled gastrointestinal condition, disease, or irregular bowel movements (including persistent diarrhea or 
(a)

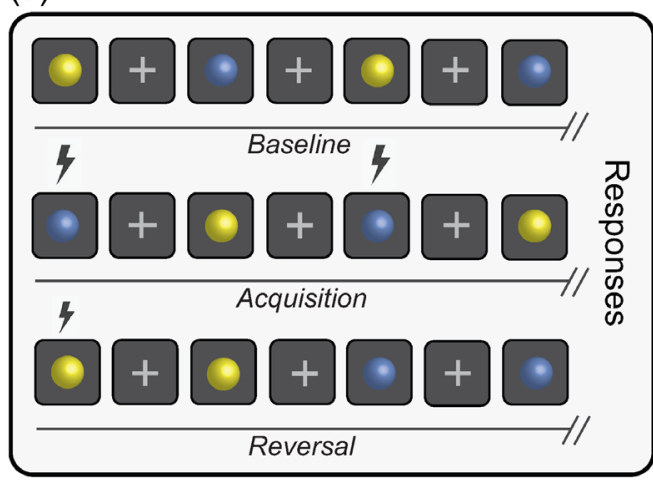

(c)
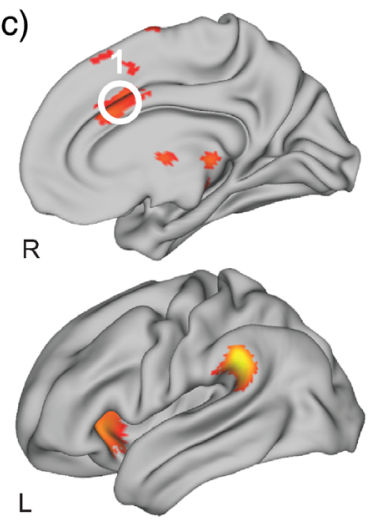

3.5 (b)

Anxious arousal
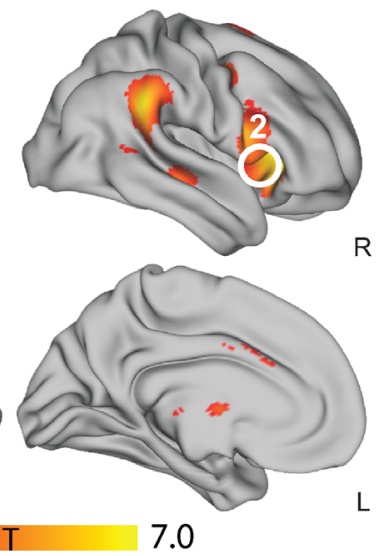

$\mathrm{R}$

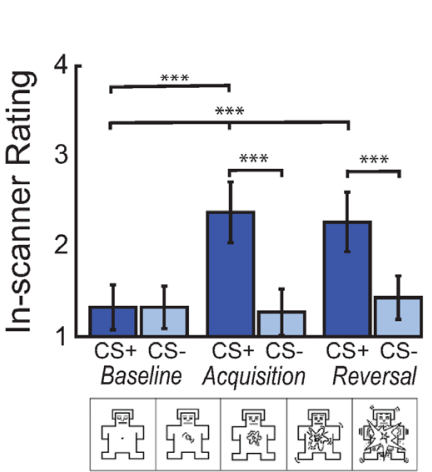

(d)
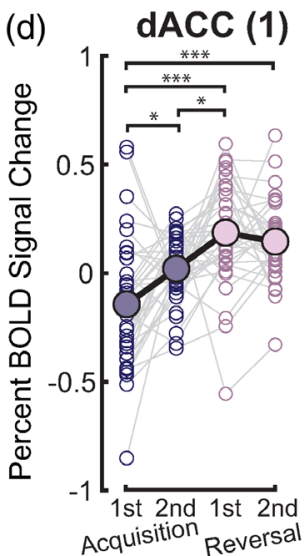
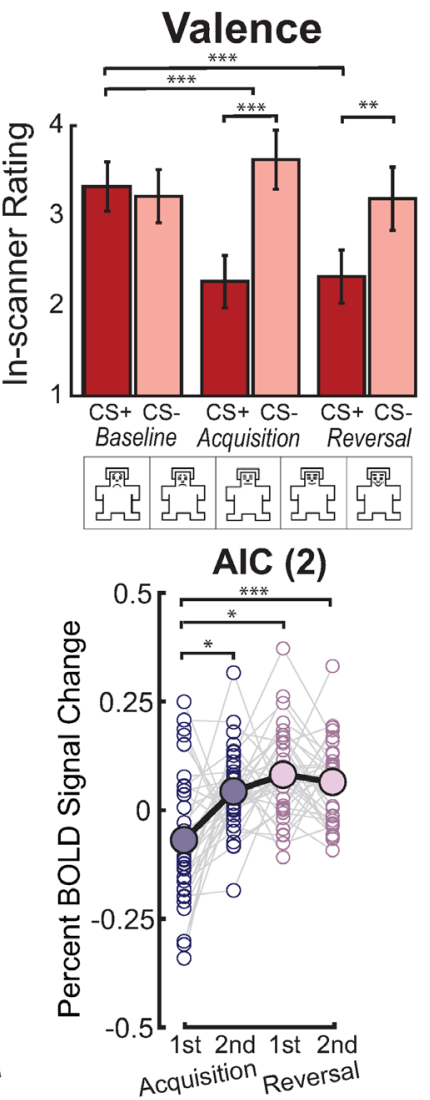

FIGURE 1 Behavioural results and neural correlates of threat acquisition and reversal. (a) Design of the threat processing paradigm implemented in the MRI scanner. The fMRI task lasted for $17 \mathrm{~min}$ and had three phases: baseline (top row), acquisition (middle row), and reversal (bottom row). Blue and yellow spheres were used as the conditioned stimuli (CS). Between each CS presentation, a white fixation cross appeared which served as a fixed interstimulus interval (ISI). The unconditioned stimulus (US, lightning bolt) was an aversive auditory burst. During acquisition, the US co-terminated with one of the CS (forming a threat, CS + ) and not with the other (forming a safety, CS-). During reversal, the pairing of the US and CS was switched. Immediately after each phase (in-scanner, as a continuation of the task phase), participants were asked to rate the spheres in terms of subjective bodily anxiety sensations and affective valence on a five-point Likert scale (Self-Assessment Manikins, SAM; Bradley and Lang, 1994). (b) Behavioural results for subjective in-scanner ratings of the threat and safety signals during baseline, acquisition and reversal for bodily anxiety sensations (left) and affective valence (right). The ratings confirmed the differential aversiveness of the threat relative to the safety signal, and the acquisition/reversal compared to the baseline stimulus (where no US was present). For post-hoc $t$ tests (Bonferroni-corrected), ${ }^{* *}$ denotes $p_{\mathrm{FWE}}<0.001$ and ${ }^{* * *}$ denotes $p_{\mathrm{FWE}}<0.0001$. (c) The contrast overall (combining acquisition and reversal task phases) threat $(\mathrm{CS}+)>$ safety $(\mathrm{CS}-)$ was associated with significant $\left(p_{\mathrm{FWE}}<0.05\right.$ at cluster level, high threshold of $\left.p_{\text {uncorrected }}<0.001\right)$ group level activation in cortical and subcortical brain regions, including the mid dACC (white circle ' 1 ') and right AIC (white circle ' 2 '; details in Table S1). (d) The difference in mean percent BOLD signal change responses between threat and safety signals were assessed in the dACC and AIC during acquisition (first and second half) and reversal (first and second half). Solid dots and black lines represent the group-level mean percent BOLD signal change responses. For post hoc paired $t$ tests (Bonferroni-corrected), ${ }^{*}$ denotes $p_{\mathrm{FWE}}<0.05$, and ${ }^{* * *}$ denotes $p_{\mathrm{FWE}}<0.0001$

constipation); history of psoriasis or recurrent eczema; major changes to dietary intake in the past month (self-report); consumption of $\geq 5$ standard alcoholic drinks per day; recreational drug use $\geq 1$ occasion in the past 3 months; acute disease at time of enrolment; pregnancy or lactation; and use of the following medications within the past 3 months: antibiotics, antifungals, antivirals, antiparasitics, corticosteroids, cytokines, methotrexate or immunosuppressive cytotoxic agents, large doses of commercial probiotics, or selective serotonin reuptake inhibitors. Gastrointestinal and microbiota-related exclusion criteria were adapted from an existing framework provided by The Human Microbiome Project Consortium (Methé et al., 2012). Dietary assessments, including the Traditional Mediterranean diet (TMD) questionnaire, reported gastrointestinal symptoms, current medication use, and recent changes to major food groups was administered by an Accredited Practising Dietitian (APD). Each participant completed: (i) a health and neurocognitive assessment; (ii) home collection of a stool sample and (iii) structural (T1) and functional magnetic resonance (MR) scans. Study requirements were separated into two sessions; the second session completed no longer than 14 days after the first (4.8 \pm 3.9 days between sessions, mean $\pm \mathrm{SD}$ ).

\section{2 | Experimental paradigm}

A validated framework to study threat learning and updating is a Pavlovian threat processing paradigm, wherein an emotionally neutral 
stimulus (CS) is differentially conditioned with an aversive auditory event (US) before the contingency is subsequently switched in a later reversal phase. The fMRI task, previously used by Savage et al. (2020a), lasted for $17 \mathrm{~min}$ and had three phases: baseline, acquisition, and reversal (Figure 1a). Blue and yellow spheres, presented for $2 \mathrm{~s}$ against a black background, were used as the conditioned stimuli (CS). Between each CS presentation, a white fixation cross appeared, which served as a fixed inter-stimulus interval (ISI, $12 \mathrm{~s}$ ). The unconditioned stimulus (US) was an aversive auditory (white noise) burst (50 ms) presented at 75-100 dB that occurred at the end of CS+ presentation and co-terminated with the CS. The US volume was determined during a pre-task calibration, where participants were asked to rate the unpleasantness/averseness of the white noise. The white noise burst serving as the US has been validated in previous $\mathrm{FMRI}$ studies (Harrison et al., 2017; Savage et al., 2020a). During baseline, the CS were each presented five times and no US occurred. During acquisition, the US co-terminated with one of the CS (forming a CS+) and not with the other (forming a CS-, safety). The colour of the CS+ was counterbalanced across subjects and the CS-US pairing occurred one third of the time, enabling the classification of CS + unpaired trials and the subsequent analysis of threat responses without US confounding. During the reversal phase, the pairing of the US and CS was switched. 10 presentations of the CS+ unpaired, five of the CS+ paired and 10 presentations of the CS- occurred during both acquisition and reversal task phases, with no more than two consecutive trials of the same stimuli. Throughout the paper, the CS + unpaired is simply referred to as threat (initial threat for the acquisition phase and updated threat for the reversal phase) for ease of readability. Immediately after each phase (in-scanner, as a continuation of the task phase), participants were asked to rate the spheres in terms of anticipatory anxiety and emotional valence on a five-point Likert scale (Self-Assessment Manikins, SAM; Bradley and Lang, 1994). Upon completion of each task phase, participants were instructed to respond to questions assessing subjective anticipatory anxiety ("How anxious did the [blue or yellow] sphere make you feel?" Responses ranged from 1 = 'not at all anxious' to 5 = 'very anxious'); and emotional valence ("How unpleasant/pleasant did you find the [blue or yellow] sphere?" Responses ranged from 1 = 'very unpleasant' to 5 = 'very pleasant'). Responses were made using a hand-held buttonpress box held in the participant's dominant hand. Prior to the scan, participants were familiarised with the scales, response box, and the volume of the US.

\section{3 | Image acquisition and pre-processing}

Images were acquired on a 3 T Siemens Prisma MR scanner equipped with a 64-channel head coil. For the fMRI task, whole brain echoplanar images were acquired using a multiband sequence (multiband factor of 8, GRAPPA factor of 1). 1227 volumes were collected with the following parameters: voxel size $=2 \mathrm{~mm}^{3}$; TR $=810 \mathrm{~ms}$; $\mathrm{TE}=30 \mathrm{~mm} ;$ flip angle $=53^{\circ} ; \mathrm{FOV}=212 \mathrm{~mm}$; slice thickness $=2 \mathrm{~mm} ; 72$ slices; $0.63 \mathrm{~ms}$ echo spacing. 11 and spin echo (anterior to posterior and posterior to anterior directions) images were also acquired to assist with pre-processing of the functional data. Image pre-processing was performed using fMRIPrep version 1.3.2 (Esteban et al., 2019) and Python scripts developed in-house (available online: https://zenodo.org/record/3556980\#.XIZUr6j7TIU). Briefly, pre-processing involved head-motion correction, susceptibility distortion correction, confounds estimation, coregistration, and regression of nuisance covariates including WM, CSF, and the six head motion parameters. Smoothing using a FWHM Gaussian filter $(10 \mathrm{~mm})$ was performed with the SPM12 software (Wellcome Trust Centre for Neuroimaging, UK).

\subsection{Stool collection}

Participants were provided with a stool collection kit customised for this study and were advised to collect and return the stool sample within 24-48 $\mathrm{h}$ before/after the MR scan. We advised participants that a failure to return the stool sample within the required timeframe would result in their exclusion from the study. No individuals were excluded at this stage. The kit included a stool nucleic acid collection and preservation tube (Norgen Biotek Corp., Thorold, Ontario, Canada), written instructions on how and when to collect the stool sample, and a pair of plastic gloves. Upon return, each stool sample was labelled with a de-identified participant code, and transported and stored in $\mathrm{a}-80^{\circ} \mathrm{C}$ freezer until sample processing.

\section{5 | DNA preparation and 16S rRNA gene sequencing}

Tissue homogenization was performed using tubes containing $1.4 \mathrm{~mm}$ ceramic beads (Precellys Lysing Kit). DNA was extracted from samples and quantitated using Nanodrop 2000 (Thermo Scientific). PCR amplification was performed on the V3-V4 hypervariable region of the $16 \mathrm{~S}$ rRNA gene, and sequenced on a MiSeq sequencer (Australian Genome Research Facility, Brisbane). Paired-end reads were joined using PEAR v0.9.6 and PCR primer sequences were removed using Cudadapt. Sequence data were processed using Quantitative Insights Into Microbial Ecology (QIIME) software suite v1.9.1 using default settings. The raw read length was 300 base pairs. Of the total reads generated, 87$90 \%$ of raw reads had a quality score $>30$. The average number of reads per sample was 81,524 (range: 13,028-134,069). USEARCH v8.0 was used to cluster the sequences into Operational Taxonomic Units (OTUs) using the identity threshold of $97 \%$. Only OTUs with at least two reads were included. Representative sequences of each OTU were taxonomically classified using USEARCH, and aligned to the Greengenes reference alignment (v13.8) using PyNAST. The OTU table was normalised (total sum scaling) and square root transformed to account for the non-normal distribution of taxonomic count data. Samples were rarefied to a read depth of 5511 for diversity analyses. For $\alpha$-diversity indices, we calculated Chao1, Shannon, Simpson and Inverse Simpson measures. For our high level assessment linking 
microbiota to brain, we opted to use Inverse Simpson diversity due to its large inter-individual variability. The functional capacity of each sample was predicted using a computational modelling approach, called Phylogenetic Investigation of Communities by Reconstruction of Unobserved States (PICRUSt) (Langille et al., 2013). Gene counts encoding enzymes were then predicted using the metagenome_predictions.py script which were then mapped onto Kyoto Encyclopaedia of Genes and Genomes (KEGG) pathways (Kanehisa and Goto, 2000). We focused specifically on gene counts encoding terminal enzymes (i.e. final conversions) involved in SCFA production, identified using the KEGG reference pathways for butanoate metabolism (KEGG ref: 00650) and propanoate metabolism (KEGG ref: 00640). Gene counts that were not represented in at least $50 \%$ of samples were excluded from further analyses. The contributions of OTUs were predicted using the metagenome_contributions.py script, and were further summarised into percent contributions at the genus, order and phylum level. Tutorial steps outlining the full PICRUSt pipeline can be found online (http://picrust.github.io/picrust/).

\section{6 | Neuroimaging analyses}

For each participant, the pre-processed images were included in a first-level GLM analysis, performed with SPM12. For all three phases, the onsets of each CS event-type (baseline: $n=5$ per CS; acquisition and reversal: $\mathrm{CS}-$ (safety) $=10, \mathrm{CS}+$ unpaired (threat) $n=10, \mathrm{CS}$ + paired, $n=5$ ) were modelled as a series of delta (stick) functions, convoluted to the canonical haemodynamic response function (HRF). Model parameters were estimated using Restricted Maximum Likelihood (ReML). The resulting set of first-level contrast images were carried forward to group-level random-effects analysis. The difference in percent BOLD signal change between the threat and safety signals was extracted from the AIC and $\mathrm{dACC}$ during the acquisition and reversal task phases using the Marsbar toolbox for SPM (Brett et al., 2002). To disentangle the effects within each phase, we independently examined the differences between early (first five CS+ unpaired presentations) and late (last five CS+ unpaired presentations) mean responses in both the acquisition and reversal task phases. Neuroimaging analyses were reproduced using an alternative smoothing kernel of $5 \mathrm{~mm}$ and demonstrated consistent group-level GLM and DCM results (Figure S7 and Table S5).

\section{7 | Specification and inversion of DCMs at the first level}

We used DCM, a computational framework to investigate the effective (directed) connectivity between and within (self-connections) cortical regions. To do this, DCM for fMRI couples a bilinear model of neural dynamics with a biophysical model of haemodynamics. Details regarding this method can be found elsewhere (Friston et al., 2003). Subject-specific regions of interest (ROIs) were defined as a $5 \mathrm{~mm}$ sphere centred over each subject's peak functional activation, constrained within the functional mask generated by the group-level contrast overall (acquisition and reversal) threat > safety ( $p_{\mathrm{FWE}}$ $<0.05$ at cluster-level, high threshold of $p_{\text {uncorr }}<0.001$; Figure 1c). Subjects who had no or minimal task-related fMRI activation in AIC and $\mathrm{dACC}$ were excluded from the DCM analysis ( $p_{\text {uncorr }}<0.1$; Figures S8 and S9). Having identified the AIC ( $x=32, y=34, z=0)$ and $\mathrm{dACC}(x=2, y=20, z=28$ ) coordinates at the group level, the spatially closest (Euclidian distance) peak coordinates for each individual subject were manually located and the time series were extracted as the peak eigenvariate for all remaining participants ( $n=33$, exclusion of 5). Inspection of the ROls showed that they were all located within $10 \mathrm{~mm}$ from the group level peak (Figure S1) and were anatomically consistent with previous work (Savage et al., 2020a; Tian and Zalesky, 2018). DCM, as a hypothesis-driven framework, operates on a user-defined model space specified through the choice of: (i) endogenous (context-independent average) connections, (ii) contextual (experimental) modulation of endogenous connections and (iii) direct inputs (e.g. CS stimuli) to the system. We constructed three models, all of which considered bidirectional endogenous connections between the AIC and $\mathrm{AACC}$, and intrinsic connections at both brain regions (i.e. a fully connected A-matrix). The driving inputs (C-matrix) consisted of visual (threat/safety) and auditory (CS + paired) stimulus, modelling the possibility that input could enter at either the AIC or dACC. This allowed the US (white noise) to affect driving inputs separately from the CS+ for which there was a threat response but no auditory sound. The difference between models arises from the choice of contextual modulators (B-matrix). Specifically, the B-matrix was specified to test whether there were differential modulatory effects between the two task phases: acquisition and reversal. The first model accounted for the modulation evoked exclusively by the CS+ events during acquisition and reversal (each phase considered as a separate modulator, Figure $2 b$, Model 1 ). The second model accounts for the modulatory effects of all events (all trials of CS+, safety and ISIs) acquired during the acquisition and reversal task phases (i.e. to model tonic block effects from threat processing; Figure $2 \mathrm{~b}$, model 2 ). Finally, the third model combines the two aforementioned models, accounting for both threat-related, and blockrelated events for acquisition and reversal task phases (Figure $2 b$, Model 3). Model inversion was performed for each subject using the DCM12 routines implemented in SPM12. Bayesian model selection with random effects analysis was used to select the most likely model given the data (as assessed by the highest exceedance probability).

\section{8 | Second-level parametric empirical Bayes analysis}

The winning DCM model was brought forward to a second-level Parametric Empirical Bayes (PEB) analysis to investigate where the mean endogenous (task-independent) and modulatory effects were expressed, as well as assessing inter-subject, microbiome-associated variability in responses. To do this, the PEB scheme begins by collating the estimated parameters of interest from all subjects, including the 


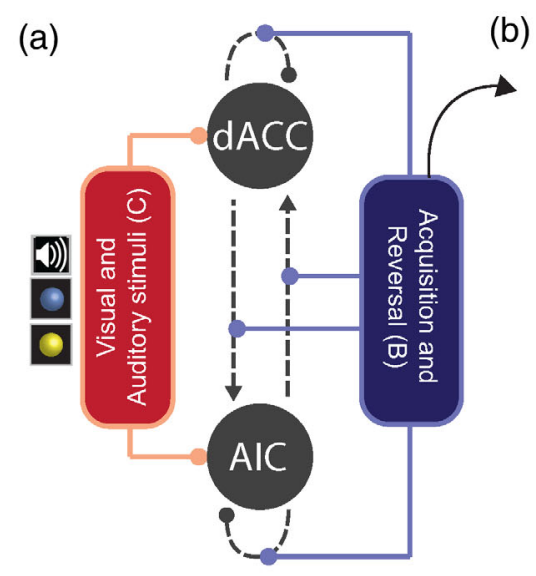

(b)

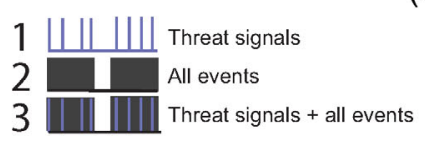

Bayesian Model Selection

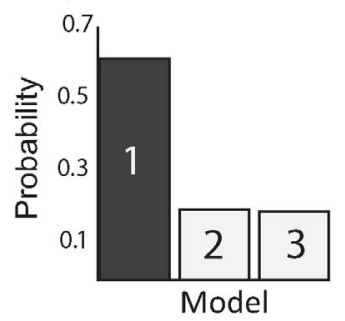

(c)

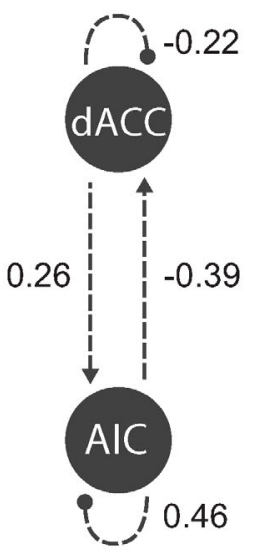

(d)
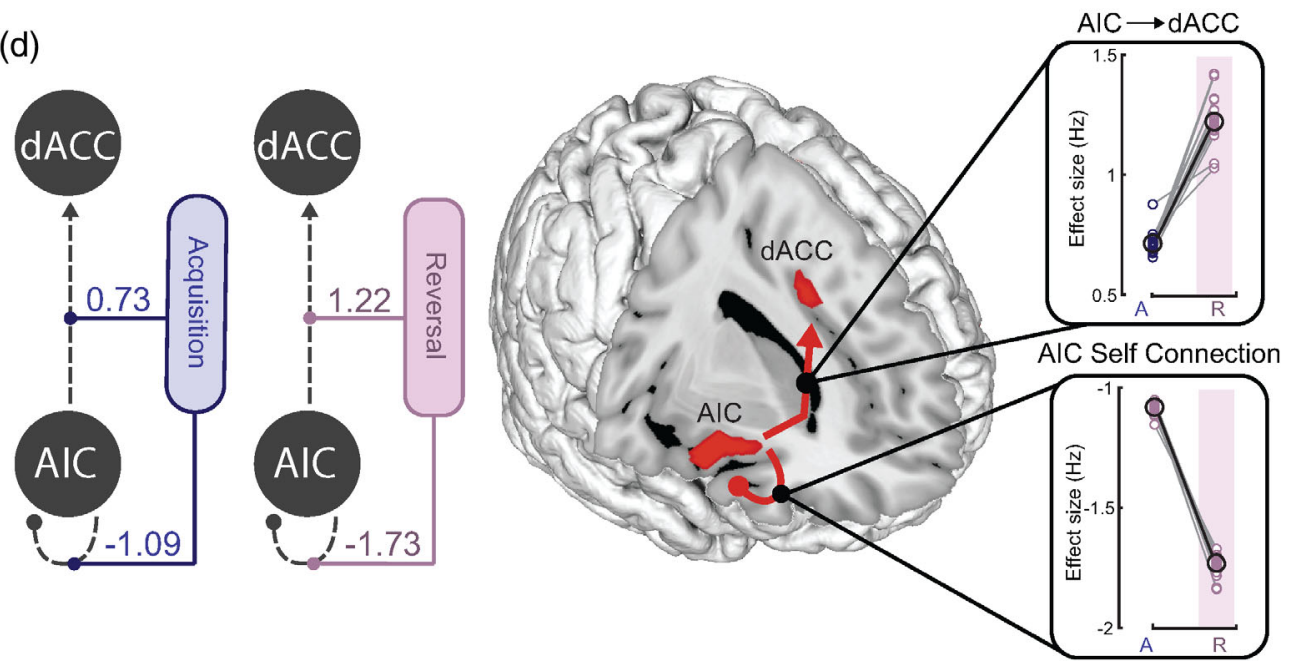

FIG URE 2 Neural dynamics supporting threat learning and updating. (a) Specification of the DCM model space in terms of: (i) taskindependent effective connectivity (grey, dashed lines) (A-matrix); (ii) modulatory connections (B-matrix) (blue), including threat signals in both acquisition and reversal task phases; and (iii) direct inputs to the system (C-matrix) comprising visual (all CS events) and auditory (US) stimuli (red). (b) Three models were estimated for each subject (see text for details). The difference between models arises from the specification of contextual modulators (threat signals, all trials, or both). Bayesian Model Selection showed that the exclusive modulation by threat signals (Model 1) best explained the fMRI data (as accessed by the highest exceedance probability). (c) Results from Bayesian Model Reduction (BMR) on second level Parametric Empirical Bayes analysis of trial-independent (fixed) connections across individuals. Results showed a positive modulation from the $\mathrm{dACC}$ to the AIC, a negative modulation from the AIC to the $\mathrm{dACC}$, and local effects within both regions. (d) BMR results showed a significant modulatory effect of threat signals on patterns of effective connectivity during acquisition and reversal (left). Results highlight very consistent modulatory effects of task phase (from acquisition to reversal) on AIC $\rightarrow \mathrm{dACC}$ (top, right) and AIC self-connections (bottom, right)

expected values of the parameters, their covariance matrices and approximate likelihoods. Next, Bayesian model comparison was performed using these first level model parameters to infer group effects. The group mean was modelled by including a constant term in the design matrix. Bayesian model reduction over the second level parameters was subsequently performed, which performs a greedy search over all parameters, and prunes away those that do not contribute to the model's log evidence (Friston et al., 2016). With an understanding of the group-level effects, we then performed a second PEB, this time specifying a design matrix with three regressors. The first regressor modelled the group mean, and the second and third modelled the microbiota covariates (orthogonalised and mean-centred). High-level microbial measures including the ratio between Bacteroidetes/ Firmicutes $(B / F)$, and $\alpha$-diversity-were selected a priori based on previous work (Ley et al., 2006; Mariat et al., 2009; Lozupone et al., 2012). We note that unlike previous work (Ley et al., 2006), we did not observe a significant relationship between $B / F$ ratio and body mass index (BMI; $R=-0.21, p=0.25$ ). PEB returns each parameter, where covariate-specific and group means are reported in terms of the expected values (Ep) and their corresponding posterior probabilities (Pp). Consistent with (Zeidman et al., 2019), Pps > 0.95 were considered to have a nonzero effect.

\section{$2.9 \quad$ Enterotype clustering}

To perform the brain-microbiota assessment at genus-level, we reduced the dimensionality of the microbiota into features that 
represented the greatest sources of variability in the data. Microbiota samples were clustered into enterotypes using methods previously described in Arumugam et al. (2011), which are readily available online (http://enterotype.embl.de/enterotypes.html). We used the CalinskiHarabasz $(\mathrm{CH})$ index (Caliński and Harabasz, 1974) to identify the optimal number of clusters (from $k=2$ to $k=20$ ). The $\mathrm{CH}$ index is used as a measure of clustering performance, representing the ratio of the sum of between-clusters dispersion to inter-cluster dispersion for all clusters. A higher $\mathrm{CH}$ index represents more well-defined and separated clusters:

$$
\mathrm{CH}_{\mathrm{k}}=\frac{\frac{\mathrm{B}_{k}}{k-1}}{\frac{W_{k}}{n-k}}
$$

where $B_{k}$ is the between-cluster sum of squares (i.e. the squared distances between all samples that are not in the same cluster), and $W_{k}$ is the within-cluster sum of squares (i.e. the squared distances between all samples that are from the same cluster), and $n$ is the number of samples. The number of clusters, $k$, was selected based on the maximum $\mathrm{CH}_{k}$ score (representing a higher ratio between betweencluster distances to within-cluster distances). Additionally, the silhouette index (SI; Rousseeuw, 1987) was adopted to assess how similar a sample was to its own cluster (cohesion), compared to other clusters (range of -1 to +1 , where a high positive value indicates that samples are well aligned to its own cluster). The $\mathrm{SI}$ is based on the Euclidean distance between points in the same cluster, compared to the distance of points of different clusters:

$$
S(i)=\frac{b(i)-a(i)}{\max (a(i), b(i))}
$$

where $a(i)$ is the average dissimilarity (or distance) of sample i to all other samples in the same cluster, while $b(i)$ is the average dissimilarity (or distance) to all objects in the closest other cluster. Betweenclass analysis (BCA) was performed using the R package ade4, and provided a visualization of the clusters and the taxonomic drivers for the enterotypes (Figure S2a). Specifically, the genera that most influenced the partitioning of samples into the three enterotypes were identified based on the top relative abundance in each enterotype (cluster).

\subsection{0 | Co-abundance network construction}

Using the relative abundances at genus-level (total sum scaled and square root transformed), we computed the Pearson correlation coefficients between the four enterotype-driving genera (Bacteroides, Ruminococcus, Oscillospira, and Prevotella) and all other genera to generate an undirected, weighted co-occurrence network. Positive (cooccurrence) and negative (co-exclusion or competitive) relationships, using cut-offs at $>0.39$ and $<-0.39$, respectively, were visualised using the interactive platform Gephi (Version 0.9.2), using the force atlas template (Bastian et al., 2009). To confirm the robustness of network interactions, we also computed Sparcc correlations (Friedman and Alm, 2012) on count data collapsed at genus level (Figure S6).

\subsection{1 | Multiple regression analysis}

Multiple linear regression analysis was used to test the association between AIC-dACC responses with the relative abundance of each driving genera. To avoid overfitting the models, we used PCA to reduce the dimensionality of each of the normalised (z-scored) sets of brain measures. For PCA inputs, we used the DCM modulatory connections for threat conditioning (four parameters) and reversal (four parameters), as well as the percent signal change values for both task phases in the AIC (four parameters) and AACC (four parameters). If the first PCA explained $<50 \%$ variance, the second PCA component was also included. This resulted in seven PCA components $(2 \times$ threat conditioning; $1 \times$ threat reversal; $2 \times$ AIC percent BOLD signal change; $2 \times \mathrm{dACC}$ percent BOLD signal change) representing our 16 brain measures (Figure S4).

\subsection{2 | Sparse canonical correlation analysis}

$I_{1}$-norm regularised sparse canonical correlation analysis (sCCA) was implemented using default settings in the R package, "PMA" (Witten et al., 2009). This approach has been designed to partially alleviate modelling challenges in small sample sizes by introducing a penalty for the elements of the weight vectors (Wang et al., 2020). The aim of this analysis is to reduce the variable set according to their most important directions of linear variation, while allowing the microbiotabrain associations to be interpreted within the original variable space. In this implementation, the tuning parameter was automatically selected for each dataset (microbiota and brain) using a permutation scheme $(n=10,000$ ), repeated across 10 different candidate sparsity parameters (ranging from 0.1 to 0.7 ). The best penalty for each dataset was selected based on the highest z-statistic, and the sCCA was then repeated using this parameter (Figure S5a). Leave-one-out (LOO) cross-validation was performed to confirm the absence of any single subject outliers (Figure S5b). A secondary cross-validation was performed by randomly removing $15 \%$ of the total sample (permutations $=1000$ ) and assessing the stability of the (a) sCCA correlation and (b) brain and microbiota weights (Figure S5b). An overview of the complete preprocessing and analysis pipeline is shown in Figure S10.

\section{3 | RESULTS}

\section{1 | Behavioural results}

Participants completed a differential threat conditioning task that involved initial learning ("acquisition phase") of threat (CS+) and safety (CS-) signal associations, and subsequent updating of these associations during a "reversal phase" (Savage et al., 2020a; Figure 1a). At the 
end of each task phase (including a baseline phase), participants rated the extent to which the threat and safety signals evoked bodily anxiety sensations, respectively, as well as their affective valence. Consistent with previous work (Savage et al., 2020a), results from the participants' subjective in-scanner ratings confirmed that the threat signals induced significantly $(p<0.001)$ higher bodily anxiety sensations (anxious arousal) and were more unpleasant (negative valence) than the safety signals during both acquisition and reversal phases, as well as compared to the threat signals during baseline (pre-conditioning, where no US was present; Figure 1b, Data S1). As expected, there were no significant differences in participants' subjective ratings of the threat and safety signals at baseline (Figure $1 \mathrm{~b}$ and Data S1). Results from two-factor repeated measures ANOVAs and post-hoc paired $t$ tests are reported in Data S1.

\subsection{Computations within specialised brain regions}

As participants demonstrated successful differential learning across both the acquisition and reversal task phases, we examined the general neural correlates of threat learning by averaging responses to the threat and safety signals across these phases. That is, we assessed brain regions showing higher activity to the threat signals during acquisition and reversal, when compared to the safety signal. We selected the right $\mathrm{AIC}$ and mid $\mathrm{AACC}$ as our regions of interest (Table S1 and Figure S1). The right AIC (opposed to left) has more robustly been linked to the awareness and representation of interoceptive visceral cues, providing a specific neural substrate to assess gut-brain interactions (Critchley et al., 2002; Critchley et al., 2004). While sub-regions of the cingulate and prefrontal regions (e.g. subgenual ACC, ventromedial prefrontal cortex) are also suggested to support important and distinct aspects of threat and safety learning, the dACC has specifically been described as the "viseromotor" interoceptive cortex, having a more selective role in integrating internal bodily signals to regulate our responses to threat (Harrison et al., 2015; Fullana et al., 2016). As expected, our results showed a robust group-level difference in the right AIC and mid AACC $\left(p_{\mathrm{FWE}}<0.05\right.$ at cluster-level, cluster isolated using $p_{\text {uncorr }}<0.001$, Figure 1c), consistent with previous work using this task (Savage et al., 2020a, b). Additional brain regions, including the middle frontal gyrus, and ventral striatum were also more active in the overall (initial and reversed) threat > safety contrast (Table S1). While this contrast reflects the joint neural correlates of acquisition and reversal phases, we also examined the specificity of AIC and AACC activation patterns over time (comparing acquisition to reversal). To do this, we extracted the difference in mean fMRI percent signal change responses between threat and safety signals relative to the baseline of the AIC and $\mathrm{dACC}$ (regions defined by two $5 \mathrm{~mm}$ spheres using the peak maxima from the overall contrast; Brett et al., 2002). In line with previous work using a similar task (Schiller et al., 2008), we separately assessed brain responses during the early (first half) and late (second half) acquisition and reversal phases to examine putative learning-related changes (Brett et al., 2002). Results of a one-way repeated-measures ANOVA showed a global difference across conditions for both dACC $\left(F_{3,99}=13.99, p=1.12 \times 10^{-7}, \eta_{p}{ }^{2}=0.24\right)$ and $\operatorname{AIC}\left(F_{3,99}=3.01\right.$, $p=0.03, \eta_{p}{ }^{2}=0.07$; Figure $1 \mathrm{~d}$ ). In the dACC, post hoc paired t-tests (Bonferroni-corrected) showed a significant increase in response from early acquisition to late acquisition $\left(t_{33}=-2.89, p_{\mathrm{FWE}}=0.04\right)$, and from acquisition to reversal (early acquisition vs. early reversal, $t_{33}$ $=-4.63, p_{\mathrm{FWE}}=3.34 \times 10^{-4}$; early acquisition vs. late reversal, $t_{33}$ $=-4.84, p_{\mathrm{FWE}}=1.75 \times 10^{-4}$; late acquisition vs. early reversal, $t_{33}$ $=-3.27, p_{\mathrm{FWE}}=0.02$; Figure $1 \mathrm{~d}$ ). In the AIC, an increase in response was observed in the transition from early acquisition to late acquisition ( $t_{33}=-3.43, p_{\mathrm{FWE}}=0.01$ ) and early acquisition to reversal (early acquisition vs. early reversal, $t_{33}=-4.14, p_{\mathrm{FWE}}=0.001$; early acquisition to late reversal, $t_{33}=-4.46, p_{\mathrm{FWE}}=5.30 \times 10^{-4}$; Figure $1 \mathrm{~d}$ ). Stronger shifts in $\mathrm{dACC}$ and $\mathrm{AIC}$ responses were captured between task phases, rather than between early and late divisions within each phase.

\section{3 | Neural dynamics supporting threat processing}

We used Dynamic Causal Modelling (DCM, see Section 2) to study neural interactions between the AIC and dACC. We estimated three model variants for each subject, assuming bidirectional connections, modulatory effects on all possible connections, and allowing direct inputs at both nodes (Figure 2a). The three models occupied distinct functional pathways that could be altered by threat and/or all taskrelated events (Figure 2a,b). More specifically, the first model tested for modulatory effects evoked exclusively by the threat signals during acquisition and reversal (two regressors, as each phase was considered as a separate modulator, Figure $2 \mathrm{~b}$, Model 1 ). The second model accounted for the modulatory effects of all trials occurring during the acquisition and reversal phases, including threat, safety, and interstimulus intervals (two regressors, Figure $2 b$, Model 2). The third model combined the two aforementioned models, accounting for both threat-related, and trial-related events for acquisition and reversal (four regressors, Figure $2 b$, Model 3 ). This approach allowed us to assess whether directed connectivity strengths within the AIC-dACC network were modulated exclusively by threat-related stimuli, or whether a "tonic-like" effect exists (responses to threat produces a constant or increasing modulatory response over the duration of the task).

Bayesian model selection with random effects indicated the first model (threat-based signals) as the most likely given the data (as assessed by exceedance probability; Figure 2b). To identify significant connections within this model at the group-level, we applied PEB. This uses Bayesian model reduction (BMR) to automatically search over all parameters and prune effects that do not meaningfully contribute to the model's log evidence. Significant effects are here defined by parameters where the posterior probability $(\mathrm{Pp})$ of a nonzero effect was $\geq 0.95$. We started by assessing connection strengths independent of task activation. These results showed that across all trials there was a positive connection from the dACC to the AIC, an 
inhibitory connection from the AIC to the $\mathrm{AACC}$, and local responses at the AIC and $\mathrm{dACC}$ that were consistent across individuals (Figure 2c). We next tested the effects of threat acquisition and reversal on all potential connections (AIC to dACC, dACC to AIC, AIC and $\mathrm{dACC}$ self-connections). In the acquisition phase, the threat-based signals enhanced bottom-up connectivity from the AIC to dACC, as well as local inhibitory responses at the AIC (Figure $2 \mathrm{~d}$, blue). The same pattern of results was observed during threat reversal, but with stronger effects overall (Figure 2d, pink). The expected values (Ep) and Pps for all parameters are presented in Table S2.

\section{4 | $\alpha$-Diversity covaries with AIC-dACC dynamics}

Addressing the first of our microbiota-brain aims, we investigated whether patterns of effective (modulatory) connections underpinning threat learning and updating covaried with high-level microbial properties. Emerging evidence suggests that high-level microbial measures, including the $B / F$ ratio and $\alpha$-diversity (a joint measure of the richness and evenness of microorganisms), are important and distinct indicators of the promotion and maintenance of host health (Ley et al., 2006; Mariat et al., 2009; Lozupone et al., 2012). As there was sufficient variability in these measures across individuals, the $\mathrm{B} / \mathrm{F}$ ratio and $\alpha$-diversity constituted two group-level parametric covariates (Figure 3a,b). To test for an effect of these covariates on directed patterns of connectivity, we again applied the Parametric Empirical Bayes method-this time testing for the presence of microbial covariation within the neural data. We assessed different combinations of covariates: group-level effects, a single microbiota covariate, or both microbiota covariates (Figure 3c). Results indicated that the most likely model included the second microbiota covariate, $\alpha$-diversity (Model 3 , posterior probability of 0.96 , Figure $3 c$ ). To confirm the validity of these findings, we repeated the analyses on surrogate models generated by permuting values within the microbiota covariates. The posterior probability of our winning model was significantly higher compared to the random surrogate models ( $n=1000$ permutations; Figure $3 d$ ). To isolate the significant parameters contributing to the model evidence, that is specific connections that covaried with $\alpha$-diversity, we applied BMR on the winning model (Figure 3e). Inter-individual measures of $\alpha$-diversity covaried with regulatory (inhibitory) control from the dACC to AIC, and local inhibitory responses at the AIC during threat reversal (Figure $3 \mathrm{e}, \mathrm{f}$ ). A confirmatory analysis further suggested that stronger signalling from the $\mathrm{dACC}$ to the AIC during reversal downregulates AIC activity (inhibitory modulation; Data S2). To control for common confounds often associated with human microbiota-based research, we adopted the relevant exclusion criteria from The Human Microbiome Project Consortium (Methé et al., 2012) and ensured that faecal samples were collected within a 48-h window before or after the scanning session (see Section 2). Furthermore, a confirmatory analysis controlling for the effects of sex and age showed highly consistent results.

\subsection{The human microbiota exhibits variability of enterotype-driving genera}

We next investigated whether similar patterns of microbiota-brain interactions emerged at lower levels of the taxonomic hierarchy (i.e. from coarse-level measures to genus level). To reduce the dimensionality of the microbiota into features that represent the greatest sources of variability in the data, we performed an enterotype analysis which uses the partitioning around medoids (PAM; Kaufman and Rousseeuw, 2009) clustering (Data S3 and Figure S2). In line with previous work (Arumugam et al., 2011), between-class analysis revealed that clusters were driven by interindividual variability in the following genera (Falony et al., 2016): Bacteroides (enterotype 1), Ruminococcus/Oscillospira (enterotype 2), and Prevotella (enterotype 3; Figure S2). While clusters were nonrandom (Data S3 and Figure S3), they were also not exclusively discrete. Instead, visualization of the samples suggested that the data were distributed along a continuum (Figure S2). Based on these findings, our subsequent analyses focused on a dimensional assessment of the link between the four discriminate genera and brain dynamics. This approach was also motivated by previous work, suggesting an interrogation of dominant taxa that drive separation between samples, rather than the enterotype classifications themselves, as features to link with clinical or behavioural variables (Gorvitovskaia et al., 2016; Cheng and Ning, 2019).

\subsection{Distinct effects of genus abundance on threat-processing brain dynamics}

We first assessed whether AIC-dACC network strengths were linearly correlated with the abundance of each driving genus independently. Specifically, separate multiple linear regressions were used to assess the association between the abundance of each candidate genus (Bacteroides, Oscillospira, Ruminococcus, and Prevotella) with distinct brain indices (threat-induced connectivity strengths and percent BOLD signal change). To reduce the dimensionality and improve interpretability, a principal components analysis (PCA) was separately applied to the values of (a) AIC-dACC connectivity during threat acquisition; (b) AIC-dACC connectivity during threat reversal; and percent BOLD signal change responses in the (c) AIC; and (d) dACC. When the first principal component (PC) for each brain set (a-d) explained $<50 \%$ variance, the second component was also included. This resulted in seven PCs $(2 \times$ threat acquisition, $1 \times$ threat reversal, $2 \times \mathrm{AIC}$, and $2 \times \mathrm{dACC}$ percent signal change) which together constituted our regression features (Figure S4). PCs representing brain variables explained a significant amount of the variance in Ruminococcus $\left(R^{2}=0.49, F_{[25,33]}=3.48, p=0.01\right.$, Figure $\left.4 a\right)$. Ruminococcus abundance yielded three significant regression weights $\left(\beta=-0.47, t_{(25)}\right.$ $=-2.65, p=0.01 ; \beta=-0.37, t_{(25)}=-2.43, p=0.02 ; \beta=0.45, t_{(25)}$ $=2.71, p=0.01$ ), supporting an interaction with connectivity responses during threat acquisition (first PCA for threat acquisition), threat reversal (first PCA for threat reversal) and local activity in the 
(a)

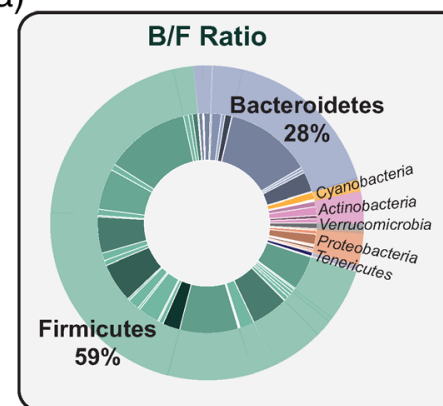

Design Matrix

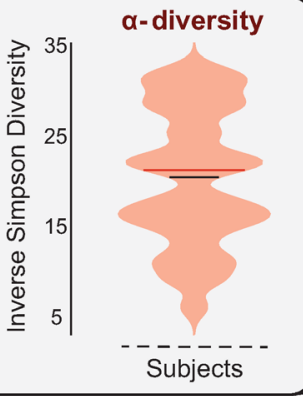

(b)

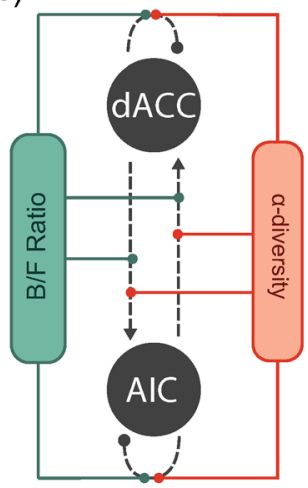

(c)

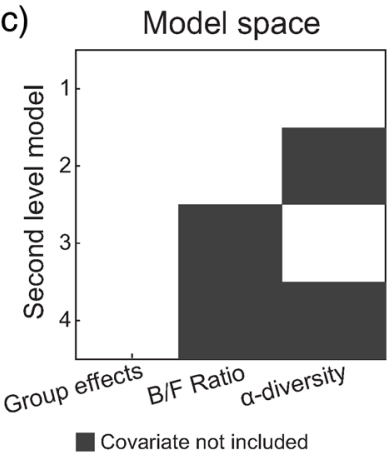

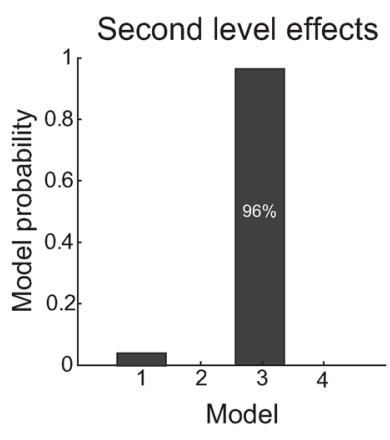

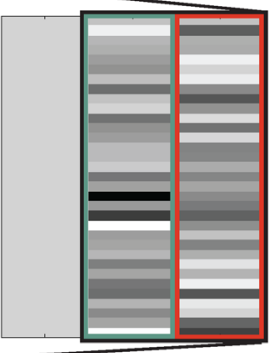

(d)

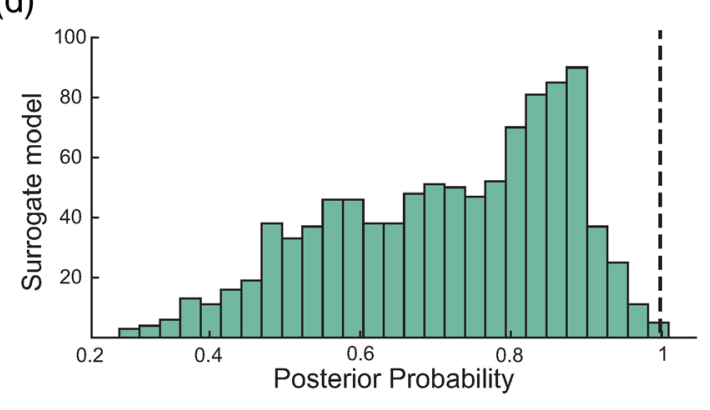

(e)

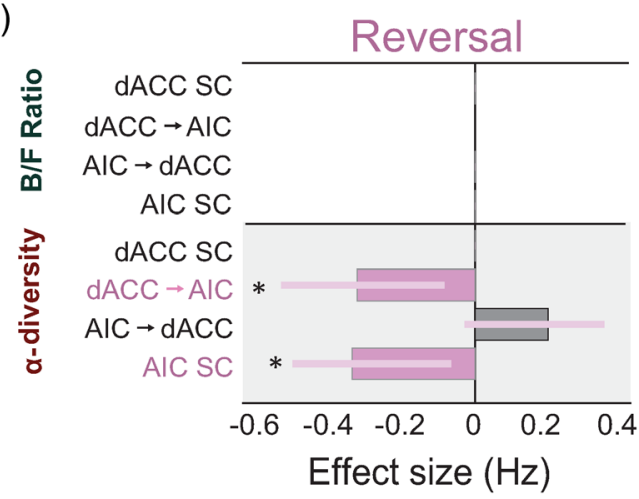

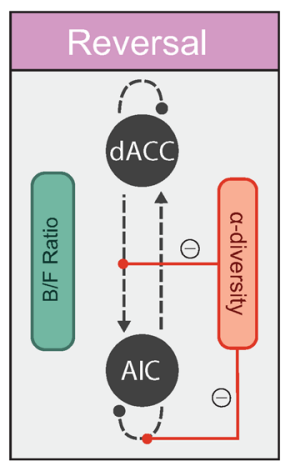

(f)

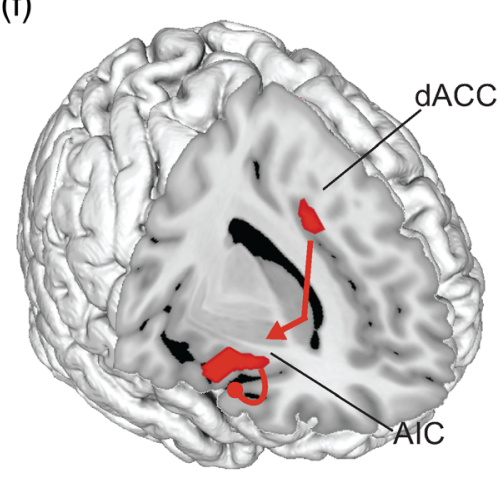

FIGURE 3 Effects of high-level microbial properties on threat learning and updating. (a) Inlet (left) shows the mean proportions of B/F, and the violin plot (middle) shows the distribution of $\alpha$-diversity (Inverse Simpson diversity) scores in our samples $(n=38)$. These two microbiota features constituted our two regressors in the Parametric Empirical Bayes model (design matrix columns two and three, right). The first column models the group mean. (b) Specification of the model space showing all possible modulatory connections where the microbiota features can interact within the AIC-dACC network. (c) Model space (left) showing possible second level models (including a null model), where both covariates (Model 1), one covariate (Models 2 and 3), or no covariates (Model 4, null) contribute to the model evidence. The winning second level model (right) included the second covariate (Model 3), at a posterior probability (Pp) of 0.96. (d) Distribution of Pp results from surrogate testing. Dashed black line indicates the $\mathrm{Pp}(0.96)$ of the winning model for the original (nonpermuted) data. (e) Results from Bayesian Model Reduction (BMR) showing the effect sizes (expressed in $\mathrm{Hz}$ ) of modulatory connections associated with $\alpha$-diversity during threat reversal. Significant parameters are those with a $\mathrm{Pp}>0.95$, indicated with an asterisk. The length of the bars corresponds to the expected probability (Ep) and the error bars are $90 \%$ Bayesian confidence intervals. SC represents self-connections. (f) Anatomical representation showing the significant modulatory connections associated with $\alpha$-diversity during the threat reversal phase

dACC (first PCA for dACC percent BOLD signal change), respectively. While the overall regression model was not significant for Bacteroides $\left(R^{2}=0.37, F_{[25,33]}=2.12, p=0.08\right.$; Figure $\left.4 \mathrm{~b}\right)$ or Oscillospira $\left(R^{2}\right.$ $=0.33, F_{[25,33]}=1.74, p=0.15$, Figure $4 c$ ), there were individual variables that were significant. For Bacteroides, the model yielded one significant regression weight $\left(\beta=0.40, t_{(25)}=-2.80, p=0.01\right)$, suggesting an association with connectivity responses during threat acquisition (represented by first PCA for threat acquisition). Oscillospira abundance was significantly associated with local activity in the dACC (first PCA for dACC percent BOLD signal change; $\left.\beta=0.52, t_{(25)}=-2.81, p=0.01\right)$. Variance in Prevotella $\left(R^{2}=0.25, F_{[25,33]}=1.16, p=0.36\right.$; Figure $\left.4 d\right)$ was not related to any specific set of brain measures. Multiple regression results are reported in full in Table S4. A confirmatory analysis controlling for 
(a)

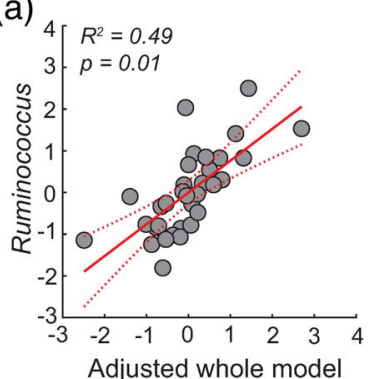

(b)

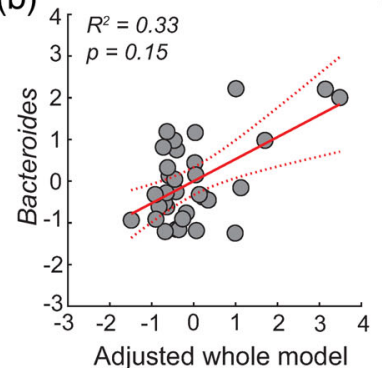

(c)

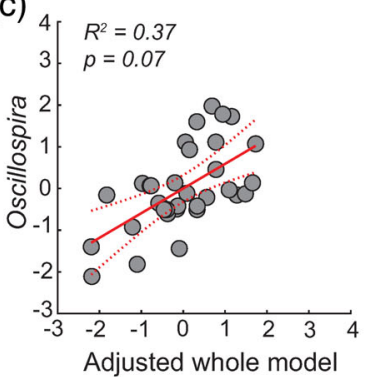

(d)

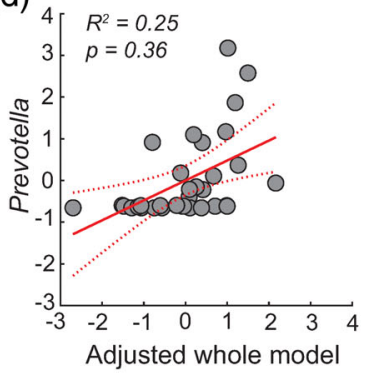

(e)

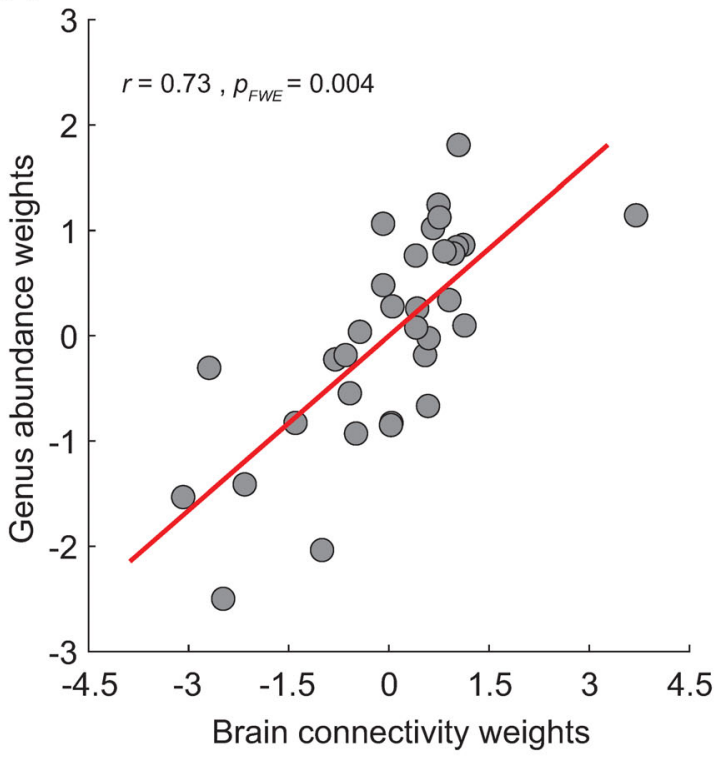

(f)

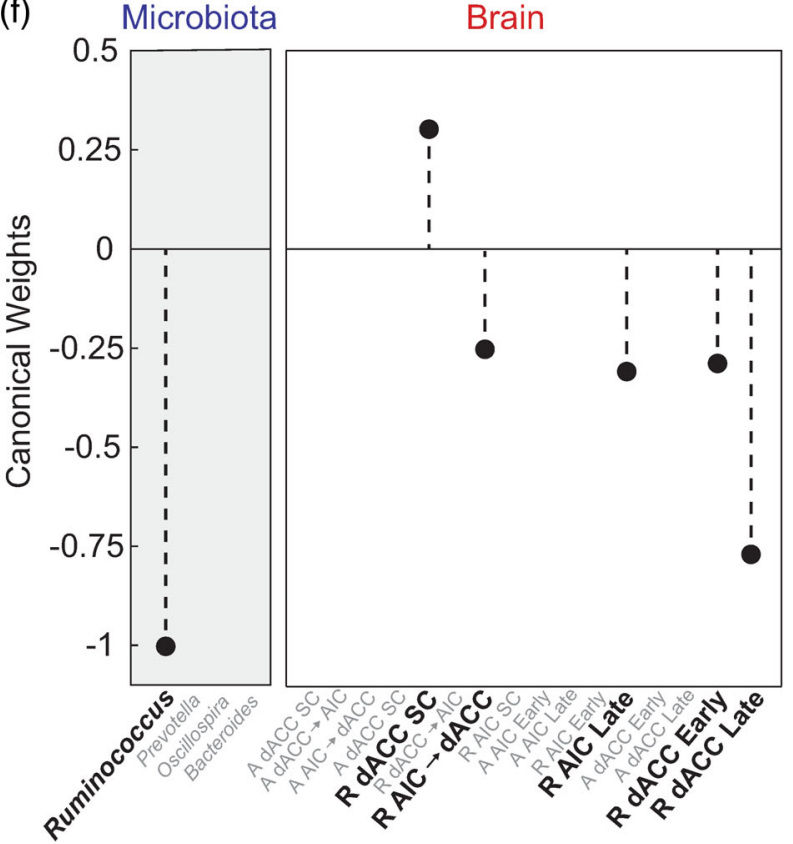

FIGURE 4 Associations between the driving genera and threat-related brain processes. Principal components analysis (PCA) was used to reduce the dimensionality of brain variables, which resulted in seven PCs representing connectivity strengths during threat learning (task acquisition phase), threat reversal (task reversal phase), and percent BOLD signal change responses at the AIC and dACC. Results from four independent multiple regressions showed that brain responses predicted the relative abundance of Ruminococcus (a). While there were individual regression weights that were significant for (b) Bacteroides and (c) Oscillospira, the overall regression model was not significant. Variance in (d) Prevotella was not related to any specific set of brain measures. 95\% confidence intervals are represented by the dashed red lines. (e) Multivariate analysis (sparse CCA, sCCA) showed a single significant mode of population covariation linking threat processing measures of brain activity and effective connectivity with Ruminococcus abundance. (f) Bold text shows microbiome and brain weights (coefficients) contributing to the sCCA. Features in grey text represent zero-contributing features to the sCCA, as imposed by the $I_{1}$-norm penalty term. Brain variables prefixed with an ' $A$ ' refer to those occurring in the task acquisition phase, ' $R$ ' refers to brain variables in the task reversal phase, and ' $S C$ ' refers to modulatory self-connections

the effects of sex and age showed highly consistent results $\left(R^{2}=0.49\right.$, $\left.F_{[25,33]}=3.48, p=0.01\right)$.

\section{7 | Multivariate association between driving genera and threat processing brain dynamics}

We next investigated the hypothesis that the microbiota and AICdACC activity exhibit a multivariate relationship-that is multiple dimensions along the genus axes could map to multivariate brain patterns. This hypothesis is motivated by the fact that driving genera are unlikely to exert independent effects on the neural substrates of threat processing. Results from a sCCA showed a single mode of population variation linking threat-related brain responses with the driving genera (Figure $4 \mathrm{e},(r)=0.73, p_{\mathrm{FWE}}=0.004$, see Section 2). This analysis identified a significant canonical mode that associated increased Ruminococcus abundance with stronger feedforward connectivity from the AIC to $\mathrm{AACC}$, and local activity within the AIC and $\mathrm{dACC}$ during threat reversal (Figure 4f). The stability of the significant sCCA was supported by a LOO cross-validation analysis. This analysis involved performing 32 sCCAs using the same sparsity parameter as the original SCCA, but with each iteration removing one subject $(r=0.73 \pm 0.01,0.69-0.77$ [mean $\pm \mathrm{SD}$, range], Figure S5). To further confirm the stability of the SCCA, we performed a secondary 
cross-validation by randomly removing $15 \%$ of the dataset (permutations $=1000)$ and assessing the stability of the CCA correlation and weights ( $r=0.74 \pm 0.03,0.61-0.82$, [mean \pm SD, range], Figure S5b).

\section{8 | Potential mechanisms linking genus abundance to threat-related brain processes}

Growing preclinical evidence supports a causal relationship between the production of SCFAs-including butyrate, propionate, and acetate-and host behaviour (Stafford et al., 2012; van de Wouw et al., 2018). Specifically, it has recently been suggested that SCFAs may be critical modulators of neuronal functions associated with threat reversal (Stafford et al., 2012) and anxiolytic effects (Burokas et al., 2017). In the light of these findings, we assessed whether the driving genera linked to threat-induced AIC-dACC network activity were major contributors to the microbial production of SCFAs. Given that microorganisms work in consort to perform and maintain metabolic functions (c.f. cross-feeding relationships [Belenguer et al., 2006;
Baxter et al., 2019]), we started by assessing how the driving genera interact with the broader microbiota ecosystem. To achieve this goal, we constructed interaction networks highlighting co-abundance (positive correlations, $r>0.39$ ) and co-exclusion (negative correlations, $r<-0.39)$ relationships between the driving genera and the wider microbial community (Figure $5 \mathrm{a}-\mathrm{c}$ ). The co-abundance networks revealed that nodes within the Oscillospira/Ruminococcus network (Figure 5a) were negatively correlated with nodes within the Bacteroides network (Figure $5 b$ ). A confirmatory network analysis using Sparcc correlations (Friedman and Alm, 2012) supported the above results (Figure S6).

We then predicted the functional capacity of each microbial sample from its $16 S$ profile using PICRUSt [Langille et al., 2013]). This algorithm predicted the functional capacity of microorganisms by using ancestral-state reconstruction to estimate which gene families are present and how they interact to form the composite metagenome. Functional predictions were then mapped onto the Kyoto Encyclopaedia of Genes and Genomes (KEGG; Kanehisa and Goto, 2000) to isolate the gene content encoding for terminal
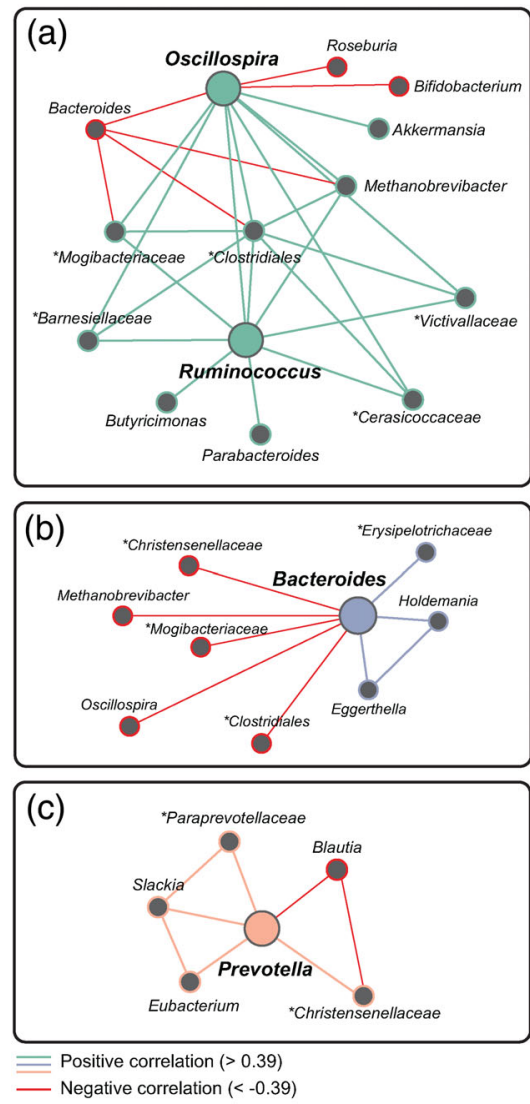

(d)

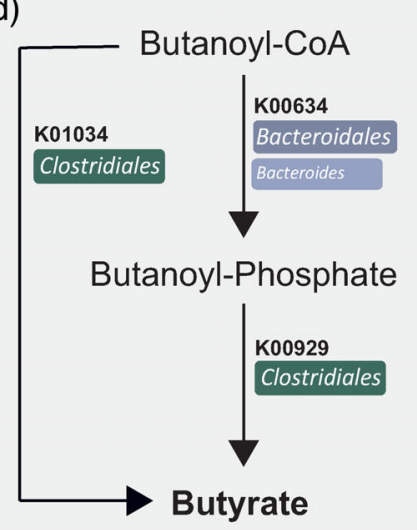

Propanoyl-CoA

Propanoyl-Phosphate

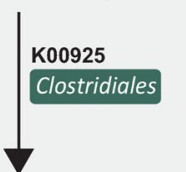

Propionate

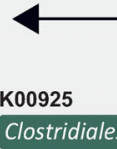

K01026 Clostridiales Ruminococcus
Oscillospira

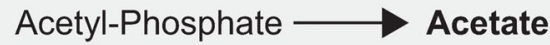

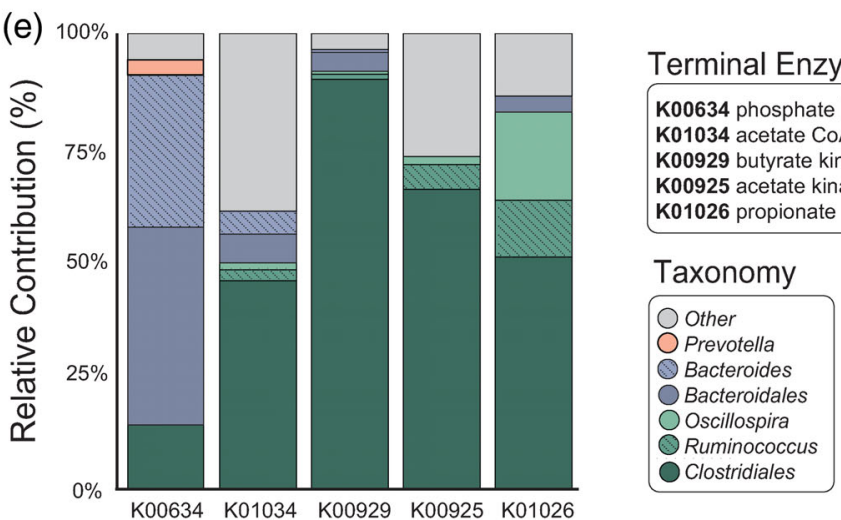

FIGURE 5 Link between microbiota genera associated to threat-related brain processes and functional pathways supporting the production of short-chain fatty acids. Co-occurrence and co-exclusion relationships between the driving genera (large nodes) including (a) Ruminococcus/ Oscillospira, (b) Bacteroides, and (c) Prevotella. Unclassified genera are described at a broader taxonomic rank above genus level (i.e. family or order) and are marked by asterisks. Graphs are visualised as a force-directed layout using Gephi (Version 0.9.2), using the force atlas template (Bastian et al., 2009). (d) Metabolic pathways (derived from the Kyoto Encyclopaedia of Genes and Genomes pathways) representing final enzymatic conversions (terminal enzymes) involved in butyrate, propionate, and acetate production. The major contributor(s) to each gene-encoding enzyme have been identified in coloured boxes. (e) Decomposition of core/major genera and orders contributing to SCFA pathways. Dark tones represent contributions from a higher taxonomic rank: order. Hatched and lighter tones represent contributions from driving genera 
enzymes involved in the production of SCFAs (i.e. butyrate, propionate and acetate; Figure $5 \mathrm{~d}$ ). The estimated contributions (i.e. the degree to which each microorganism contributed to each sample's gene content) were represented at both the genus and order scale, and were summarised at the group level (Figure 5e). Members of the Clostridiales order, including Ruminococcus and Oscillospira, were identified as major contributors to all terminal enzymes involved in SCFA production. Other genera within the Clostridiales order, including Blautia, Lachnospira, and Coprococcus were also contributors to these pathways. Bacteroidales (order), with a substantial contribution from Bacteroides (genus) (Figure 5e), were involved in the sub-terminal reaction (K00634) in butyrate production. As showed in Figure 4e, Ruminococcus is the strongest multivariate driver of brain patterns. Crucially, results from our functional analysis highlight that the SCFA production pathways also rely on Ruminococcus and the order (Clostridiales) from which it originates. These findings are consistent with the notion that the detected microbiota features contributing to gut-brain associations supporting threat processing are linked to SCFA production, either directly or in consort with co-occurrence interactions.

\section{4 | DISCUSSION}

We used a multidisciplinary approach to assess the relationship between the human gut microbiota and a brain network supporting the subjective experience of threat processing via cognitiveinteroceptive appraisal mechanisms (Fullana et al., 2016; Harrison et al., 2015; Kalisch and Gerlicher, 2014). Results highlighted an association between microbial abundance patterns-reflected across different taxonomic scales-with neural dynamics between the AIC and $\mathrm{dACC}$ during threat learning and threat updating processes. By considering broad ecological relationships and estimated functional properties of the microbiome, we provide support for the notion that microbial genera influencing threat-related brain processes are involved in SCFA production. More broadly, our findings provide impetus to pursue future research assessing the viability of the gut microbiome to impact brain activity and behaviour linked to threatrelated disorders (Harrison et al., 2015).

High-level ecological measures are thought to recapitulate key organizational principles of the gut microbiota (Ley et al., 2006; Mariat et al., 2009; Lozupone et al., 2012). We found that inter-individual variability in $\alpha$-diversity-a joint measure of the evenness and richness of residing microorganisms-was associated with the strength of inhibitory patterns of connectivity from the dACC to the AIC, and self-inhibitory responses within the AIC during threat reversal (Figure $3 e, f)$. The reversal of learned threat stimuli associations requires an updating process, where former safety signals are reevaluated as a new threat. The $\alpha$-diversity-mediated modulation of $\mathrm{dACC}$ to AIC is consistent with previous work, suggesting that processes involving threat updating are supported by cognitive computations within the dACC (Stevens et al., 2011; Savage et al., 2020a). These findings are noteworthy, as $\alpha$-diversity is thought to engender a microbial ecosystem that is robust and resilient to environmental perturbation (Bokulich et al., 2016). Higher functional redundancy allows the microbiota to compensate for the functions of absent species, including adequate production of microbiota-derived metabolites to meet host demands (Valdes et al., 2018). Accordingly, reduced $\alpha$-diversity has been linked to exacerbated fear reactivity in infants (Aatsinki et al., 2019; Gao et al., 2019) and altered insular connectivity in adults (Curtis et al., 2019). The Bacteroidetes to Firmicutes ratio was not linked to threat-related AIC-dACC patterns of activity and connectivity. While some evidence suggests a link between this ratio and inflammation (Verdam et al., 2013), obesity (Ley et al., 2006), and chronic pain (Labus et al., 2017), the utility of phyla-level ratios as a biomarker in mental health remains debated. Findings from the current study do not support the putative link between the Bacteroidetes to Firmicutes ratio and threat-related brain processing.

While diversity measures are important from a whole systems approach, when considered in isolation it is unclear whether effects are driven by highly conserved features (i.e. common to all members of major phyla), or if they emerge within finer taxonomic scales. The analysis of genus-level composition is thought to represent an intermediate scale; striking a balance between the complexities of interpreting highlevel ecological measures, with a reductionist approach that isolates species of interest. Results from the multiple regression and SCCA analysis converge in supporting an association between Ruminococcus abundance and activity from the AIC to the $\mathrm{ACC}$, as well as local activity within the AIC and dACC during threat reversal. More broadly, Ruminococcus appears to link to key brain processes facilitating the update of previously reinforced safety signals to new threat associations (Figure 4a,f).

The biological mechanisms supporting the causal interplay between the gut microbiome and brain processes underpinning human behaviour are not yet fully understood. The microbiota is thought to impact brain activity via the production of SCFAs including acetate, propionate, and butyrate (Dalile et al., 2019). Accordingly, recent preclinical studies suggest that alterations in microbiotaderived metabolites, including SCFAs, contribute to neuronal activity and behaviour linked to threat processing (Stafford et al., 2012; Whittle and Singewald, 2014). SCFAs are thought to exert their effects on the CNS directly or indirectly through immune, endocrine, vagal, and other humoral pathways (Dalile et al., 2019). Locally, SCFAs maintain intestinal barrier integrity (Lewis et al., 2010), promote mucous production (Pelaseyed et al., 2014), and regulate the secretion of interleukins (Soliman et al., 2012), all of which reduce systemic inflammation. Proinflammatory cytokines and molecular intermediates linked to systemic inflammation have been thought to influence neuroinflammation and associated changes in mood and behaviour by crossing the blood-brain barrier (BBB; Reichenberg et al., 2001; Harrison et al., 2009; Schrepf et al., 2018). SCFAs are also signalling molecules that act as histone deacetylase inhibitors (Kratsman et al., 2016; Stilling et al., 2016), as well as endogenous ligands for Gprotein coupled receptors, free fatty acid receptor (FFAR) 2 and FFAR3 (Brown et al., 2003). These receptors are expressed on enteroendocrine cells, various immune cells, and vagal afferents (Nøhr et al., 2015; Egerod et al., 2018). While SCFAs have been shown to cross the blood brain barrier (Liu et al., 2015; Sun et al., 2016), this 
pathway is not thought to be a major route via which SCFAs exert their influence on cortical activity and related behaviour. Enteroendocrine-mediated vagal signalling is considered to be a more direct and accessible route via which SCFAs influence cortical dynamics and behaviour (Lal et al., 2001; Bonaz et al., 2018). Within enteroendocrine cells, SCFAs are also thought to promote the synthesis of important neurotransmitters, including serotonin, which can independently exert their influence on the gut-brain axis via the vagal nerve (Reigstad et al., 2015). Information from vagal afferents converge in the nucleus tractus solitarius, which can then be relayed to cortical brain regions including the AIC and the $\mathrm{AACC}$. Accordingly, associations between enzymes involved in butyrate production and the insula structure have recently been reported (Labus et al., 2017). Here, we provide evidence indicating that Ruminococcus and co-occurring taxa play an important role in the estimated production of acetate, propionate, and butyrate. This finding is consistent with previous studies showing that Ruminococcus spp. are strongly linked to the fermentation of resistant starch, and therefore may contribute to butyrate (Morrison and Preston, 2016; Baxter et al., 2019), acetate (Louis et al., 2014; Fernández et al., 2016; Koh et al., 2016), and propionate (Koh et al., 2016) production in the colon. While our findings are estimations and thus cannot be directly extrapolated, they provide a solid rationale to motivate future work directly testing the putative key role of SCFAs in the modulation of brain processes. Future work that specifically focuses on SCFAs would also support the growing body of existing preclinical and emerging human data (Burokas et al., 2017; Boehme et al., 2019; Dalile et al., 2019; Lee et al., 2020). More specifically, preclinical work suggests a link between Ruminococcus-induced increases in SCFAs and improvements in anxiety-related behaviours (Yu et al., 2020), and stress and emotional instability (Provensi et al., 2019). However, important to note is that clinical translation of this work has yielded conflicting results. A recent study in healthy men showed that colonic administration of SCFAs attenuated the cortisol response to physiological stress, but had no effect on threat learning as assessed by subjective ratings and skin conductance responses (Dalile et al., 2020).

A number of caveats need to be considered while interpreting the results from this study. Our analysis focused on a well-defined tworegion circuit consistently implicated in human threat processing (Fullana et al., 2016). Moreover, this network has recently been linked to individual differences in anxiety sensitivity-a well validated trait measure of the fear of bodily anxiety sensations (Harrison et al., 2015; Savage et al., 2020a). Future work could assess how AIC-dACC circuitry interacts with the broader network of brain regions supporting the contextual processing of threat, and its possible interactions with the microbiota. With regards to the fMRI task, while the use of a fixed ISI has been adopted by previous work (Savage et al., 2020a; Schiller et al., 2008), we acknowledge that future work may benefit from a task variant with more events and a nonfixed ISI. We attempted to minimize common confounds associated with both acute (e.g. recent medication use and dietary intake) and general lifestyle changes in microbiota composition by selecting relevant exclusion criteria provided by The Human Microbiome Project Consortium (Methé et al., 2012). Furthermore, confirmatory analyses of the PEB and genus-level assessments when controlling for the effects of sex and age showed highly consistent results. Stool sample collection and neuroimaging were also performed in close temporal proximity (within a 48-h window). However, collection of repeat faecal samples could provide a more nuanced assessment of the relationship between microbiome and brain processes. In the current study, 16S rRNA sequencing was used as this method has been demonstrated to provide sufficient resolution to characterise genus (and broader) level interactions (Rausch et al., 2019). Furthermore, previous work has demonstrated that functional assessments performed using PICRUSt are sufficiently well correlated with genomic content to yield accurate predictions in human gut microbiome samples (Langille et al., 2013). However, as this emerging field continues to develop, the importance of combining neuroimaging and behavioural datasets with higher resolution sequencing (shotgun metagenomics), and untargeted or targeted metabolomics will be critical to extend upon this work. The specificity of SCFAs and their putative mechanistic role in altering AIC-dACC dynamics will also need to be replicated in adequately powered human interventional studies. Current results provide key knowledge and motivation to invest in future targeted work assessing the link between gut features and threat-related neural dynamics.

\section{5 | CONCLUSION}

There is convincing preclinical evidence demonstrating the effect of the gut microbiota in altering brain mechanisms supporting threat processing. However, there remains a large gap in the understanding of how microbiota consortia engenders variability in neural dynamics underpinning human threat processing. Our study supports distinct interactions between microbial abundance patterns-reflected across different taxonomic scales-with neural dynamics supporting threat learning and threat updating processes in healthy individuals. While research in this field is still in its infancy, current data highlights that the assessment of the microbial milieu may provide insights into the emergence of, and vulnerability to threat-related disorders. For example, a characterisation of key microbiota features, including diversity measures and the presence/absence of microbial communities (e.g. Ruminococcus), could be used as biomarkers in the assessment of vulnerability to anxiety-related disorders. Growing interest in the academic and medical community has also coincided with a patientdriven preference for alternative or adjunct therapies (beyond medication) to manage mental illness. Personalised modulation of the microbiome by boosting or reducing microbial communities related to maladaptive brain and behavioural responses via a dietary intervention, may represent a low-risk, efficacious, and cost-effective approach to manage affective disorders.

\section{ACKNOWLEDGEMENTS}

We thank P. Sanz-Leon for her contributions to the fMRI preprocessing pipeline. We thank E. Savage, G. Whybird, and N. Koussis for their assistance with data collection, and S. Sonkusare 
for manuscript feedback. Luca Cocchi acknowledges support from the Australian National Health and Medical Research Council (NHMRC), APP1099082.

\section{CONFLICT OF INTEREST}

The authors declare no conflict of interest.

\section{AUTHOR CONTRIBUTIONS}

Conceptualization and methodology, Caitlin V. Hall, Ben J. Harrison, Rosalyn J. Moran and Luca Cocchi; Formal analysis: Caitlin V. Hall, Kartik K. Iyer, Hannah S. Savage, Martha Zakrzewski, Rosalyn J. Moran and Luca Cocchi; Data curation: Caitlin V. Hall and Lisa A. Simms; Resources, Graham Radford-Smith, Lisa A. Simms, Luca Cocchi; Writing-original draft, review, and editing, all authors; Funding acquisition, Luca Cocchi; Supervision, Rosalyn J. Moran and Luca Cocchi.

\section{DATA AVAILABILITY STATEMENT}

Raw sequences are available online (NCBI, accession number: PRJNA766867). Functional and structural MRIs are available upon reasonable request from the corresponding author.

\section{ORCID}

Caitlin V. Hall (D) https://orcid.org/0000-0001-7013-6446 Luca Cocchi (D) https://orcid.org/0000-0003-3651-2676

\section{REFERENCES}

Aatsinki, A.-K., Lahti, L., Uusitupa, H.-M., Munukka, E., Keskitalo, A., Nolvi, S., ... Karlsson, L. (2019). Gut microbiota composition is associated with temperament traits in infants. Brain, Behavior, and Immunity, 80, 849-858.

Allman, J. M., Tetreault, N. A., Hakeem, A. Y., Manaye, K. F., Semendeferi, K., Erwin, J. M., ... Hof, P. R. (2010). The von Economo neurons in frontoinsular and anterior cingulate cortex in great apes and humans. Brain Structure and Function, 214, 495-517.

Arumugam, M., Raes, J., Pelletier, E., Le Paslier, D., Yamada, T., Mende, D. R., ... Meta, H. I. T. C. (2011). Enterotypes of the human gut microbiome. Nature, 473, 174-180.

Azzalini, D., Rebollo, I., \& Tallon-Baudry, C. (2019). Visceral signals shape brain dynamics and cognition. Trends in Cognitive Sciences, 23, 488-509.

Bastian, M., Heymann, S., Jacomy, M., 2009. Gephi: An open source software for exploring and manipulating networks. Third International AAAI Conference on Weblogs and Social Media.

Baxter, N. T., Schmidt, A. W., Venkataraman, A., Kim, K. S., Waldron, C., \& Schmidt, T. M. (2019). Dynamics of human gut microbiota and shortchain fatty acids in response to dietary interventions with three fermentable fibers. mBio, 10, e02566-e02518.

Belenguer, A., Duncan, S. H., Calder, A. G., Holtrop, G., Louis, P., Lobley, G. E., \& Flint, H. J. (2006). Two routes of metabolic crossfeeding between Bifidobacterium adolescentis and butyrate-producing anaerobes from the human gut. Applied and Environmental Microbiology, 72, 3593-3599.

Boehme, M., van de Wouw, M., Bastiaanssen, T. F. S., OlavarríaRamírez, L., Lyons, K., Fouhy, F., ... Cryan, J. F. (2019). Mid-life microbiota crises: Middle age is associated with pervasive neuroimmune alterations that are reversed by targeting the gut microbiome. Molecular Psychiatry, 25, 2567-2583.
Bokulich, N. A., Chung, J., Battaglia, T., Henderson, N., Jay, M., Li, H., ... Chen, Y. (2016). Antibiotics, birth mode, and diet shape microbiome maturation during early life. Science Translational Medicine, 8, $343 r a 382$.

Bonaz, B., Bazin, T., \& Pellissier, S. (2018). The vagus nerve at the interface of the microbiota-gut-brain axis. Frontiers in Neuroscience, 12, 49-49.

Bradley, M. M., \& Lang, P. J. (1994). Measuring emotion: The selfassessment manikin and the semantic differential. Journal of Behavior Therapy and Experimental Psychiatry, 25, 49-59.

Bravo, J. A., Forsythe, P., Chew, M. V., Escaravage, E., Savignac, H. M., Dinan, T. G., ... Cryan, J. F. (2011). Ingestion of Lactobacillus strain regulates emotional behavior and central GABA receptor expression in a mouse via the vagus nerve. Proceedings of the National Academy of Sciences, 108, 16050-16055.

Brett, M., Anton, J.-L., Valabregue, R., \& Poline, J.-B. (2002). Region of interest analysis using the MarsBar toolbox for SPM 99. Neurolmage, 16, 5497.

Brown, A. J., Goldsworthy, S. M., Barnes, A. A., Eilert, M. M., Tcheang, L., Daniels, D., ... Fraser, N. J. (2003). The Orphan G protein-coupled receptors GPR41 and GPR43 are activated by propionate and other short chain carboxylic acids. Journal of Biological Chemistry, 278, 11312-11319.

Burokas, A., Arboleya, S., Moloney, R. D., Peterson, V. L., Murphy, K., Clarke, G., ... Cryan, J. F. (2017). Targeting the microbiota-gut-brain axis: Prebiotics have anxiolytic and antidepressant-like effects and reverse the impact of chronic stress in mice. Biological Psychiatry, 82, 472-487.

Caliński, T., \& Harabasz, J. (1974). A dendrite method for cluster analysis. Communications in Statistics: Theory and Methods, 3, 1-27.

Cheng, M., \& Ning, K. (2019). Stereotypes about enterotype: The old and new ideas. Genomics, Proteomics \& Bioinformatics, 17, 4-12.

Chu, C., Murdock, M. H., Jing, D., Won, T. H., Chung, H., Kressel, A. M., ... Artis, D. (2019). The microbiota regulate neuronal function and fear extinction learning. Nature, 574, 543-548.

Clarke, G., Grenham, S., Scully, P., Fitzgerald, P., Moloney, R. D., Shanahan, F., ... Cryan, J. F. (2013). The microbiome-gut-brain axis during early life regulates the hippocampal serotonergic system in a sexdependent manner. Molecular Psychiatry, 18, 666-673.

Craig, A. D. (2009). How do you feel: now? The anterior insula and human awareness. Nature Reviews. Neuroscience, 10, 59-70.

Critchley, H. D., Mathias, C. J., \& Dolan, R. J. (2002). Fear conditioning in humans: The influence of awareness and autonomic arousal on functional neuroanatomy. Neuron, 33, 653-663.

Critchley, H. D., Wiens, S., Rotshtein, P., Öhman, A., \& Dolan, R. J. (2004). Neural systems supporting interoceptive awareness. Nature Neuroscience, 7, 189-195.

Cryan, J. F., \& Dinan, T. G. (2012). Mind-altering microorganisms: The impact of the gut microbiota on brain and behaviour. Nature Reviews Neuroscience, 13, 701-712.

Curtis, K., Stewart, C. J., Robinson, M., Molfese, D. L., Gosnell, S. N., Kosten, T. R., ... Salas, R. (2019). Insular resting state functional connectivity is associated with gut microbiota diversity. European Journal of Neuroscience, 50, 2446-2452.

Dalile, B., van Oudenhove, L., Vervliet, B., \& Verbeke, K. (2019). The role of short-chain fatty acids in microbiota-gut-brain communication. Nature Reviews Gastroenterology \& Hepatology, 16, 461-478.

Dalile, B., Vervliet, B., Bergonzelli, G., Verbeke, K., \& van Oudenhove, L. (2020). Colon-delivered short-chain fatty acids attenuate the cortisol response to psychosocial stress in healthy men: A randomized, placebo-controlled trial. Neuropsychopharmacology, 45, 2257-2266.

Desbonnet, L., Clarke, G., Traplin, A., O'Sullivan, O., Crispie, F., Moloney, R. D., ... Cryan, J. F. (2015). Gut microbiota depletion from early adolescence in mice: Implications for brain and behaviour. Brain, Behavior, and Immunity, 48, 165-173.

Egerod, K. L., Petersen, N., Timshel, P. N., Rekling, J. C., Wang, Y., Liu, Q., ... Gautron, L. (2018). Profiling of $G$ protein-coupled receptors in vagal 
afferents reveals novel gut-to-brain sensing mechanisms. Molecular Metabolism, 12, 62-75.

Esteban, O., Markiewicz, C. J., Blair, R. W., Moodie, C. A., Isik, A. I., Erramuzpe, A., ... Gorgolewski, K. J. (2019). fMRIPrep: A robust preprocessing pipeline for functional MRI. Nature Methods, 16, 111-116.

Falony, G., Joossens, M., Vieira-Silva, S., Wang, J., Darzi, Y., Faust, K., ... Raes, J. (2016). Population-level analysis of gut microbiome variation. Science, 352, 560-564.

Fernández, J., Redondo-Blanco, S., Gutiérrez-del-Río, I., Miguélez, E. M., Villar, C. J., \& Lombó, F. (2016). Colon microbiota fermentation of dietary prebiotics towards short-chain fatty acids and their roles as antiinflammatory and antitumour agents: A review. Journal of Functional Foods, 25, 511-522.

Forsythe, P., Bienenstock, J., \& Kunze, W. A. (2014). Vagal pathways for microbiome-brain-gut axis communication. Advances in Experimental Medicine and Biology, 817, 115-133.

Friedman, J., \& Alm, E. J. (2012). Inferring correlation networks from genomic survey data. PLoS Computational Biology, 8, e1002687.

Friston, K. J., Harrison, L., \& Penny, W. (2003). Dynamic causal modelling. Neurolmage, 19, 1273-1302.

Friston, K. J., Litvak, V., Oswal, A., Razi, A., Stephan, K. E., van Wijk, B. C. M., ... Zeidman, P. (2016). Bayesian model reduction and empirical Bayes for group (DCM) studies. Neurolmage, 128, 413-431.

Fullana, M. A., Harrison, B. J., Soriano-Mas, C., Vervliet, B., Cardoner, N., Avila-Parcet, A., \& Radua, J. (2016). Neural signatures of human fear conditioning: An updated and extended meta-analysis of $\mathrm{fMRI}$ studies. Molecular Psychiatry, 21, 500-508.

Gao, W., Salzwedel, A. P., Carlson, A. L., Xia, K., Azcarate-Peril, M. A., Styner, M. A., ... Knickmeyer, R. C. (2019). Gut microbiome and brain functional connectivity in infants: A preliminary study focusing on the amygdala. Psychopharmacology, 236, 1641-1651.

Garfinkel, S. N., Seth, A. K., Barrett, A. B., Suzuki, K., \& Critchley, H. D. (2015). Knowing your own heart: Distinguishing interoceptive accuracy from interoceptive awareness. Biological Psychology, 104, 65-74.

Gorvitovskaia, A., Holmes, S. P., \& Huse, S. M. (2016). Interpreting Prevotella and Bacteroides as biomarkers of diet and lifestyle. Microbiome, 4, 15.

Harrison, B. J., Fullana, M. A., Soriano-Mas, C., Via, E., Pujol, J., MartinezZalacain, I., ... Cardoner, N. (2015). A neural mediator of human anxiety sensitivity. Human Brain Mapping, 36, 3950-3958.

Harrison, B. J., Fullana, M. A., Via, E., Soriano-Mas, C., Vervliet, B., Martinez-Zalacain, I., ... Cardoner, N. (2017). Human ventromedial prefrontal cortex and the positive affective processing of safety signals. Neurolmage, 152, 12-18.

Harrison, N. A., Brydon, L., Walker, C., Gray, M. A., Steptoe, A., \& Critchley, H. D. (2009). Inflammation causes mood changes through alterations in subgenual cingulate activity and mesolimbic connectivity. Biological Psychiatry, 66, 407-414.

Kaelberer, M. M., Buchanan, K. L., Klein, M. E., Barth, B. B., Montoya, M. M., Shen, X., \& Bohórquez, D. V. (2018). A gut-brain neural circuit for nutrient sensory transduction. Science, 361, eaat5236.

Kalisch, R., \& Gerlicher, A. M. (2014). Making a mountain out of a molehill: On the role of the rostral dorsal anterior cingulate and dorsomedial prefrontal cortex in conscious threat appraisal, catastrophizing, and worrying. Neuroscience and Biobehavioral Reviews, 42, 1-8.

Kanehisa, M., \& Goto, S. (2000). KEGG: Kyoto encyclopedia of genes and genomes. Nucleic Acids Research, 28, 27-30.

Kano, M., Dupont, P., Aziz, Q., \& Fukudo, S. (2018). Understanding neurogastroenterology from neuroimaging perspective: A comprehensive review of functional and structural brain imaging in functional gastrointestinal disorders. Journal of Neurogastroenterology and Motility, 24, 512-527.

Kaufman, L., \& Rousseeuw, P. J. (2009). Finding groups in data: An introduction to cluster analysis. Hoboken, NJ: John Wiley \& Sons.
Koh, A., De Vadder, F., Kovatcheva-Datchary, P., \& Bäckhed, F. (2016). From dietary fiber to host physiology: Short-chain fatty acids as key bacterial metabolites. Cell, 165, 1332-1345.

Kratsman, N., Getselter, D., \& Elliott, E. (2016). Sodium butyrate attenuates social behavior deficits and modifies the transcription of inhibitory/excitatory genes in the frontal cortex of an autism model. Neuropharmacology, 102, 136-145.

Labus, J. S., Hollister, E. B., Jacobs, J., Kirbach, K., Oezguen, N., Gupta, A., ... Mayer, E. A. (2017). Differences in gut microbial composition correlate with regional brain volumes in irritable bowel syndrome. Microbiome, 5, 49.

Lal, S., Kirkup, A. J., Brunsden, A. M., Thompson, D. G., \& Grundy, D. (2001). Vagal afferent responses to fatty acids of different chain length in the rat. American Journal of Physiology-Gastrointestinal and Liver Physiology, 281, G907-G915.

Langille, M. G. I., Zaneveld, J., Caporaso, J. G., McDonald, D., Knights, D., Reyes, J. A., ... Huttenhower, C. (2013). Predictive functional profiling of microbial communities using 16S rRNA marker gene sequences. Nature Biotechnology, 31, 814-821.

le Poul, E., Loison, C., Struyf, S., Springael, J.-Y., Lannoy, V., Decobecq, M.E., ... van Damme, J. (2003). Functional characterization of human receptors for short chain fatty acids and their role in polymorphonuclear cell activation. Journal of Biological Chemistry, 278, 2548125489.

Lee, J., Venna, V. R., Durgan, D. J., Shi, H., Hudobenko, J., Putluri, N., ... Bryan, R. M. (2020). Young versus aged microbiota transplants to germ-free mice: Increased short-chain fatty acids and improved cognitive performance. Gut Microbes, 12(1), 1-14.

Lewis, K., Lutgendorff, F., Phan, V., Söderholm, J. D., Sherman, P. M., \& McKay, D. M. (2010). Enhanced translocation of bacteria across metabolically stressed epithelia is reduced by butyrate. Inflammatory Bowel Diseases, 16, 1138-1148.

Ley, R. E., Turnbaugh, P. J., Klein, S., \& Gordon, J. I. (2006). Human gut microbes associated with obesity. Nature, 444, 1022-1023.

Liu, J., Sun, J., Wang, F., Yu, X., Ling, Z., Li, H., ... Xu, J. (2015). Neuroprotective effects of Clostridium butyricum against vascular dementia in mice via metabolic butyrate. BioMed Research International, 2015, 412946.

Louis, P., Hold, G. L., \& Flint, H. J. (2014). The gut microbiota, bacterial metabolites and colorectal cancer. Nature Reviews Microbiology, 12, 661-672.

Lozupone, C. A., Stombaugh, J. I., Gordon, J. I., Jansson, J. K., \& Knight, R. (2012). Diversity, stability and resilience of the human gut microbiota. Nature, 489, 220-230.

Mariat, D., Firmesse, O., Levenez, F., Guimaraes, V., Sokol, H., Dore, J., ... Furet, J. P. (2009). The firmicutes/bacteroidetes ratio of the human microbiota changes with age. BMC Microbiology, 9, 123.

Mayer, E. A. (2011). Gut feelings: The emerging biology of gut-brain communication. Nature Reviews Neuroscience, 12, 453-466.

Methé, B. A., Nelson, K. E., Pop, M., Creasy, H. H., Giglio, M. G., Huttenhower, C., ... The Human Microbiome Project, C. (2012). A framework for human microbiome research. Nature, 486, 215-221.

Morrison, D. J., \& Preston, T. (2016). Formation of short chain fatty acids by the gut microbiota and their impact on human metabolism. Gut Microbes, 7, 189-200.

Neufeld, K.-A. M., Kang, N., Bienenstock, J., \& Foster, J. A. (2011). Effects of intestinal microbiota on anxiety-like behavior. Communicative \& Integrative Biology, 4, 492-494.

Nøhr, M. K., Egerod, K. L., Christiansen, S. H., Gille, A., Offermanns, S., Schwartz, T. W., \& Møller, M. (2015). Expression of the short chain fatty acid receptor GPR41/FFAR3 in autonomic and somatic sensory ganglia. Neuroscience, 290, 126-137.

Pelaseyed, T., Bergström, J. H., Gustafsson, J. K., Ermund, A., Birchenough, G. M., Schütte, A., ... Nyström, E. E. (2014). The mucus and mucins of the goblet cells and enterocytes provide the first 
defense line of the gastrointestinal tract and interact with the immune system. Immunological Reviews, 260, 8-20.

Perez-Burgos, A., Wang, B., Mao, Y.-K., Mistry, B., Neufeld, K.-A. M., Bienenstock, J., \& Kunze, W. (2012). Psychoactive bacteria Lactobacillus rhamnosus (JB-1) elicits rapid frequency facilitation in vagal afferents. American Journal of Physiology-Gastrointestinal and Liver Physiology, 304, G211-G220.

Provensi, G., Schmidt, S. D., Boehme, M., Bastiaanssen, T. F. S., Rani, B., Costa, A., ... Passani, M. B. (2019). Preventing adolescent stressinduced cognitive and microbiome changes by diet. Proceedings of the National Academy of Sciences, 116, 9644-9651.

Rausch, P., Rühlemann, M., Hermes, B. M., Doms, S., Dagan, T., Dierking, K., ... Baines, J. F. (2019). Comparative analysis of amplicon and metagenomic sequencing methods reveals key features in the evolution of animal metaorganisms. Microbiome, 7, 133.

Raybould, H. E. (2010). Gut chemosensing: Interactions between gut endocrine cells and visceral afferents. Autonomic Neuroscience, 153, 41-46.

Rebollo, I., Devauchelle, A.-D., Béranger, B., \& Tallon-Baudry, C. (2018). Stomach-brain synchrony reveals a novel, delayed-connectivity resting-state network in humans. eLife, 7, e33321.

Reichenberg, A., Yirmiya, R., Schuld, A., Kraus, T., Haack, M., Morag, A., \& Pollmächer, T. (2001). Cytokine-associated emotional and cognitive disturbances in humans. Archives of General Psychiatry, 58, 445-452.

Reigstad, C.S., Salmonson, C.E., III, J.F.R., Szurszewski, J.H., Linden, D.R., Sonnenburg, J.L., Farrugia, G., Kashyap, P.C., 2015. Gut microbes promote colonic serotonin production through an effect of short-chain fatty acids on enterochromaffin cells. The FASEB Journal 29, 13951403.

Rhee, S. H., Pothoulakis, C., \& Mayer, E. A. (2009). Principles and clinical implications of the brain-gut-enteric microbiota axis. Nature Reviews. Gastroenterology \& Hepatology, 6, 306-314.

Rousseeuw, P. J. (1987). Silhouettes: A graphical aid to the interpretation and validation of cluster analysis. Journal of Computational and Applied Mathematics, 20, 53-65.

Savage, H. S., Davey, C. G., Fullana, M. A., \& Harrison, B. J. (2020a). Clarifying the neural substrates of threat and safety reversal learning in humans. Neurolmage, 207, 116427.

Savage, H. S., Davey, C. G., Fullana, M. A., \& Harrison, B. J. (2020b). Threat and safety reversal learning in social anxiety disorder: An fMRI study. Journal of Anxiety Disorders, 76, 102321.

Schiller, D., Levy, I., Niv, Y., LeDoux, J. E., \& Phelps, E. A. (2008). From fear to safety and back: Reversal of fear in the human brain. The Journal of Neuroscience, 28, 11517-11525.

Schrepf, A., Kaplan, C. M., Ichesco, E., Larkin, T., Harte, S. E., Harris, R. E., ... Basu, N. (2018). A multi-modal MRI study of the central response to inflammation in rheumatoid arthritis. Nature Communications, 9, 2243.

Sheehan, D. V., Lecrubier, Y., Sheehan, K. H., Amorim, P., Janavs, J., Weiller, E., ... Dunbar, G. C. (1998). The mini-international neuropsychiatric interview (M.I.N.I.): The development and validation of a structured diagnostic psychiatric interview for DSM-IV and ICD-10. Journal of Clinical Psychiatry, 59(Suppl 20), 22-33.

Sherwin, E., Bordenstein, S. R., Quinn, J. L., Dinan, T. G., \& Cryan, J. F. (2019). Microbiota and the social brain. Science, 366, eaar2016.

Soliman, M. L., Smith, M. D., Houdek, H. M., \& Rosenberger, T. A. (2012). Acetate supplementation modulates brain histone acetylation and decreases interleukin-1 $\beta$ expression in a rat model of neuroinflammation. Journal of Neuroinflammation, 9, 1-14.

Stafford, J. M., Raybuck, J. D., Ryabinin, A. E., \& Lattal, K. M. (2012). Increasing histone acetylation in the hippocampus-infralimbic network enhances fear extinction. Biological Psychiatry, 72, 25-33.

Stevens, F. L., Hurley, R. A., Taber, K. H., Hurley, R. A., Hayman, L. A., \& Taber, K. H. (2011). Anterior cingulate cortex: Unique role in cognition and emotion. The Journal of Neuropsychiatry and Clinical Neurosciences, 23, 121-125.

Stilling, R. M., van de Wouw, M., Clarke, G., Stanton, C., Dinan, T. G., \& Cryan, J. F. (2016). The neuropharmacology of butyrate: The bread and butter of the microbiota-gut-brain axis? Neurochemistry International, 99, 110-132.

Sun, J., Ling, Z., Wang, F., Chen, W., Li, H., Jin, J., ... Liu, J. (2016). Clostridium butyricum pretreatment attenuates cerebral ischemia/reperfusion injury in mice via anti-oxidation and anti-apoptosis. Neuroscience Letters, 613, 30-35.

Tian, Y., \& Zalesky, A. (2018). Characterizing the functional connectivity diversity of the insula cortex: Subregions, diversity curves and behavior. Neurolmage, 183, 716-733.

Tillisch, K., Mayer, E. A., Gupta, A., Gill, Z., Brazeilles, R., Le Nevé, B., ... Labus, J. S. (2017). Brain structure and response to emotional stimuli as related to gut microbial profiles in healthy women. Psychosomatic Medicine, 79, 905-913.

Valdes, A. M., Walter, J., Segal, E., \& Spector, T. D. (2018). Role of the gut microbiota in nutrition and health. BMJ, 361, k2179.

Valles-Colomer, M., Falony, G., Darzi, Y., Tigchelaar, E. F., Wang, J., Tito, R. Y., ... Raes, J. (2019). The neuroactive potential of the human gut microbiota in quality of life and depression. Nature Microbiology, 4 , 623-632.

van de Wouw, M., Boehme, M., Lyte, J. M., Wiley, N., Strain, C., O'Sullivan, O., ... Cryan, J. F. (2018). Short-chain fatty acids: Microbial metabolites that alleviate stress-induced brain-gut axis alterations. The Journal of Physiology, 596, 4923-4944.

Verdam, F. J., Fuentes, S., de Jonge, C., Zoetendal, E. G., Erbil, R., Greve, J. W., ... Rensen, S. S. (2013). Human intestinal microbiota composition is associated with local and systemic inflammation in obesity. Obesity (Silver Spring), 21, E607-E615.

Wang, H.-T., Smallwood, J., Mourao-Miranda, J., Xia, C. H., Satterthwaite, T. D., Bassett, D. S., \& Bzdok, D. (2020). Finding the needle in a high-dimensional haystack: Canonical correlation analysis for neuroscientists. Neurolmage, 216, 116745.

Whittle, N., \& Singewald, N. (2014). HDAC inhibitors as cognitive enhancers in fear, anxiety and trauma therapy: Where do we stand? Biochemical Society Transactions, 42, 569-581.

Witten, D. M., Tibshirani, R., \& Hastie, T. (2009). A penalized matrix decomposition, with applications to sparse principal components and canonical correlation analysis. Biostatistics (Oxford, England), 10, 515-534.

Yu, L., Han, X., Cen, S., Duan, H., Feng, S., Xue, Y., ... Chen, W. (2020). Beneficial effect of GABA-rich fermented milk on insomnia involving regulation of gut microbiota. Microbiological Research, 233, 126409.

Zeidman, P., Jafarian, A., Seghier, M. L., Litvak, V., Cagnan, H., Price, C. J., \& Friston, K. J. (2019). A guide to group effective connectivity analysis, part 2: Second level analysis with PEB. Neurolmage, 200, 12-25.

\section{SUPPORTING INFORMATION}

Additional supporting information may be found in the online version of the article at the publisher's website.

How to cite this article: Hall, C. V., Harrison, B. J., Iyer, K. K., Savage, H. S., Zakrzewski, M., Simms, L. A., Radford-Smith, G., Moran, R. J., \& Cocchi, L. (2022). Microbiota links to neural dynamics supporting threat processing. Human Brain Mapping, 43(2), 733-749. https://doi.org/10.1002/hbm.25682 


\section{University Library}

\section{- M M N E R VA A gateway to Melbourne's research publications}

Minerva Access is the Institutional Repository of The University of Melbourne

Author/s:

Hall, C;Harrison, BJ;lyer, KK;Savage, HS;Zakrzewski, M;Simms, LA;Radford-Smith, G;Moran, RJ;Cocchi, L

Title:

Microbiota links to neural dynamics supporting threat processing

Date:

2021-11-23

Citation:

Hall, C., Harrison, B. J., Iyer, K. K., Savage, H. S., Zakrzewski, M., Simms, L. A., RadfordSmith, G., Moran, R. J. \& Cocchi, L. (2021). Microbiota links to neural dynamics supporting threat processing. HUMAN BRAIN MAPPING, 43 (2), pp.733-749. https://doi.org/10.1002/ hbm.25682.

Persistent Link:

http://hdl.handle.net/11343/296323

License:

CC BY-NC 